\title{
A DInSAR Investigation of the Ground Settlement Time Evolution of Ocean-Reclaimed Lands in Shanghai
}

\author{
Qing Zhao, Antonio Pepe, Member, IEEE, Wei Gao, Zhong Lu, Senior Member, IEEE, Manuela Bonano, \\ Man L. He, Jun Wang, and Xi Tang
}

\begin{abstract}
Reclaimed lands are seriously affected by ground settlement episodes, which are primarily caused by unconsolidated soils, and could result in severe damage to buildings and public infrastructures. In this work, we present a differential synthetic aperture radar (DInSAR) analysis of the ground displacement that impacts the ocean-reclaimed areas of the Nanhui New City of Shanghai (China), based on jointly exploiting persistent scatterers (PS) and small baseline (SB) approaches. The mean line of sight displacement velocity maps and the corresponding interferometric time-series of deformation were initially cross-compared to assess the accuracy of both DInSAR techniques in regions subject to long-lasting land reclamation processes. By exploiting a set of 31 SAR images collected by the ASAR/ENVISAT sensor from February 2007 to May 2010, we found the average difference between PS and SB velocity maps at $1.2 \mathrm{~mm} / \mathrm{year}$, with a root mean square difference of $5 \mathrm{~mm}$ for single measurements. Despite the increased levels of noise in the interferograms, due to the extremely time-varying electromagnetic and geometrical characteristics of the SAR scenes in correspondence to reclamation
\end{abstract}

Manuscript received September 15, 2014; revised December 11, 2014; accepted January 26, 2015. Date of publication March 17, 2015; date of current version May 26, 2015. This work has been carried out within the Dragon III ESA project ID 10644 entitled "DInSAR Detection of Surface Deformation in the Coastal Reclaimed Areas of Shanghai City." This work was supported in part by Research Grants of Science and Technology Commission of Shanghai Municipality through Project 13ZR1453900 and 13231203804, in part by HighEnd Foreign Experts Recruitment Program of the State Administration of Foreign experts Affairs through Project GDW20143100087, in part by the Fundamental Research Funds for the Central Universities, in part by Research Grant of Key Laboratory of Geographical Information Science, in part by Ministry of Education, in part by East China Normal University, through Project KLGIS2013C02, and in part by Research Grant of State Oceanic Administration Laboratory of Data Analysis and Applications through Project LDAA-2014-05. (Corresponding author: Antonio Pepe.)

Q. Zhao and W. Gao are with the Key Laboratory of Geographical Information Science, Ministry of Education, East China Normal University, Shanghai 200062, China, and also with Joint Laboratory for Environmental Remote Sensing and Data Assimilation, East China Normal University, Shanghai 200062, China (e-mail: qzhao@geo.ecnu.edu.cn; wgao419@ gmail.com).

A. Pepe and M. Bonano are with Istituto per il Rilevamento Elettromagnetico dell'Ambiente (IREA-CNR), 80124 Napoli, Italy (e-mail: pepe.a@irea.cnr.it; bonano.m@irea.cnr.it).

Z. Lu is with Roy M. Huffington Department of Earth Sciences, Southern Methodist University, Dallas, TX $752-750395$ USA (e-mail: zhonglu@smu.edu).

M. L. He and J. Wang are with Shanghai Surveying and Mapping Institute, Shanghai 200072, China (e-mail: hemanly@ hotmail.com; cug_wj@163.com).

$\mathrm{X}$. Tang is with the Key Laboratory of Geographical Information Science, Ministry of Education, East China Normal University, Shanghai 200062, China (e-mail: xtang@geo.ecnu.edu.cn).

Color versions of one or more of the figures in this paper are available online at http://ieeexplore.iee.org.

Digital Object Identifier 10.1109/JSTARS.2015.2402168 platforms, these results suggest that during first stages of reclamation processes both DInSAR methods are able to correctly identify points that preserve high level of accuracy. We have thus predicted the forthcoming time evolution of ground displacement over reclaimed platforms by combining DInSAR measurements and geotechnical-derived models, suggesting that approximately $\mathbf{9 0 \%}$ of the settlements occur within about 15 years.

Index Terms-Deformation, differential synthetic aperture radar interferometry (DInSAR), persistent scatterers (PS), small baseline (SB), time-series.

\section{INTRODUCTION}

D IFFERENTIAL synthetic aperture radar (DInSAR) interferometry [1], [2] has drawn increased attention by the scientific community over the past few years, and currently represents a well-established remote sensing technique for the continuous detection and monitoring of Earth's ground motion, with a centimeter to millimeter accuracy [3]. DInSAR is based on phase difference (interferogram) measurements between a pair of complex-valued SAR images acquired at different epochs and from slightly different orbital positions. These measurements allow for the detection of the radar line of sight (LOS) projected surface displacement component. Even though DInSAR methodology was originally applied to study single deformation episodes [4]-[7], since the development of the so-called "advanced" multitemporal DInSAR approaches, its capabilities have improved to allow for retrieval of temporal evolution of the LOS-projected displacement. Such methods are based on implementing a proper inversion of differential SAR interferogram sequences. During the past 15 years, there has been an increase in the literature written on the advancements of multitemporal DInSAR techniques, most of which analyze space-borne SAR data [8]-[15]. More recently, some experiments have also demonstrated the reliability of DInSAR measurements achieved by utilizing airborne data collected at any band [16].

Advanced multitemporal DInSAR techniques are distinguished by two approaches: the persistent scatterers (PS) [8][11] and the small baseline (SB) [12]-[15]; however, a solution that incorporates both approaches has also been proposed [17].

The PS techniques focus on analyzing point-like targets that are not significantly affected by decorrelation [18], [19], and are particularly suitable for monitoring deformation signals that impact point-wise and man-made objects, such as buildings and infrastructures in urban areas. Thus, they do not require the 
imposition of any constraints on temporal and spatial separation baselines of the used DInSAR interferograms, which are generated with respect to one common SAR scene. PS candidates are selected according to their backscattering characteristics. As a result, PS-based approaches essentially perform a modelbased, temporal phase unwrapping strategy, which relies on a two-dimensional (2-D) linear regression of the wrapped phases with respect to the average linear displacement velocity and possible digital elevation model (DEM) errors. Thus, a priori information on the displacement is mandatory, from which a linear deformation model can be established.

Conversely, the SB methods allow the investigation of displacement signals that affect distributed scatterers (DS) on the ground, making it possible to drastically increase the spatial density of DInSAR measurements, especially in semiurbanized and rural areas. However, DS targets are less impacted by decorrelation noise artifacts [18], [19], thus requiring a proper selection of a set of SAR data pairs characterized by small baseline values. Therefore, the baseline selection implies that the SAR data pairs used to produce the interferograms may be arranged in (a few) disconnected subsets that can be separated by large baselines. These subsets are independent from each other (since no suitable interferogram connecting SAR images belonging to different subsets exists), thus giving rise to an underdetermined problem for the timeseries inversion. In particular, the advanced algorithm referred to as Small BAseline Subset (SBAS) [12], which belongs to the SB class, solves this problem by searching for a minimum norm least squares (LS) solution via the singular value decomposition (SVD) method [20]. In this latter case, both multilook [12] and single-look [21] SAR interferograms can be analyzed.

Despite the differences between the PS and SB classes of algorithms, they have both been successfully used to detect and monitor deformation signals due to several natural and anthropogenic hazards, such as volcanic events, earthquakes, landslides, man-made subsiding infrastructures, and damaged buildings [22]-[37].

In this study, we present the results of a DInSAR analysis carried out over the Nanhui New City in Shanghai (China). We have relied on the application of both the advanced SBASDInSAR technique and the PS approach, with the latter relevant to the implementation provided within the interferometric point target analysis (IPTA) processing chain [9]. The major goal of the proposed analysis is to recover the temporal evolution of the displacement that occurs in ocean-reclaimed areas of Shanghai due to over pumping groundwater activities and, moreover, to consolidation processes of large areas of dredger fill and alluvial deposits [38].

Feasible measures for land reclamation from the sea to lowland countries and coastal cities have been adopted in other regions, thus resolving issues of land scarcity in highly populated areas. For instance, The Netherlands has been reclaiming land since the 16th century. During the 20th century, $1650 \mathrm{~km}^{2}$ of new land has been reclaimed for agriculture, recreation, and urban expansion [39]. Successful land reclamation has also been observed in The Palm Islands in Dubai (United Arab Emirates), populated by large man-made islands located in the Arabian Gulf [40]. Additional examples of public infrastructures built on reclaimed (or partly-reclaimed) lands [41]-[46] are: 1) Changi Airport, Singapore; 2) Hong Kong International Airport, China; 3) Kansai Airport, Japan; and 4) New Doha International Airport, Qatar, and airports in Brisbane and Sydney in Australia. In China, $1600 \mathrm{~km}^{2}$ of land was reclaimed from 2002 to 2011 [47]. An important reclamation project has just been approved by Nanhui New City (formerly Lingang New City), a coastal area of Shanghai, approximately $300 \mathrm{~km}^{2}$ in size, $45 \%$ of which was reclaimed from intertidal wetlands. The reclaimed foundation has been in a residual settlement process since the completion of the filling project. Field measurements indicate that ground settlement of the reclaimed foundation occurred during the construction of the new city. Average cumulative values of settlement from 2001 to 2006 ranged between 25 and $75 \mathrm{~mm}$ corresponding to the average annual rates between 5 and $15 \mathrm{~mm} /$ year [48].

Ground subsidence may cause serious damage to buildings, highways, airport runways, harbors, and underground facilities. Thus, the utilization of DInSAR technologies for detection and measurement of residual settlement of reclaimed lands is vital for land sustainability. Furthermore, serious land subsidence can cause a decrease in sea wall height [49], resulting in decreased protection from flooding caused by storm surges and rises in sea levels.

In this framework, the detection and continuous monitoring of ground subsidence play a key role in the assessment and mitigation of the associated risk, and provide support for decision making by local authorities. However, the extremely time-varied conditions of the SAR backscattering signals over reclaimed areas, even over short time frames, lead to severe temporal decorrelation noise artifacts in the computed interferograms. This, combined with the difficulty in finding stable point-wise scatterers in areas that, during reclamation, are semi-urbanized (or not at all during the early stages of reclamation), prevents an "easy" application of the advanced multitemporal DInSAR methodologies. New challenging problems may evolve, such as the proper identification of areas on reclaimed platforms over which interferometric phases remain (at best) less corrupted by noise, thus not affecting the accuracy level of attainable DInSAR products [33], [50]. We have thus performed a cross-comparison analysis between the independently computed SBAS and IPTA deformation DInSAR products (i.e., mean deformation velocity maps and deformation time-series), obtained by processing SAR images collected from 2007 to 2010, by the ASAR sensor onboard the ENVISAT platform. It is worth stating that, even though various independent validation studies demonstrated and assessed the retrieval accuracy of the DInSAR-based deformation timeseries, very few intercomparison analyses between the two families of PS and SB techniques have previously been attempted [51]-[53]. Furthermore, those that had been conducted were over extended regions and did not specifically focus on those regions subject to severe and long-lasting reclamation processes.

The PS-IPTA/SBAS time-series of deformation computed in correspondence to the location of a properly identified set of high quality points have now been used, in conjunction 


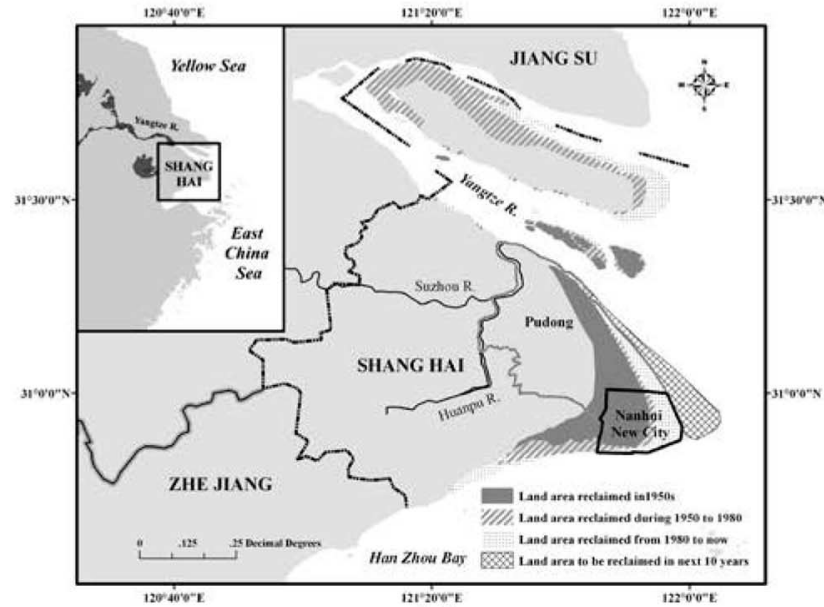

(a)

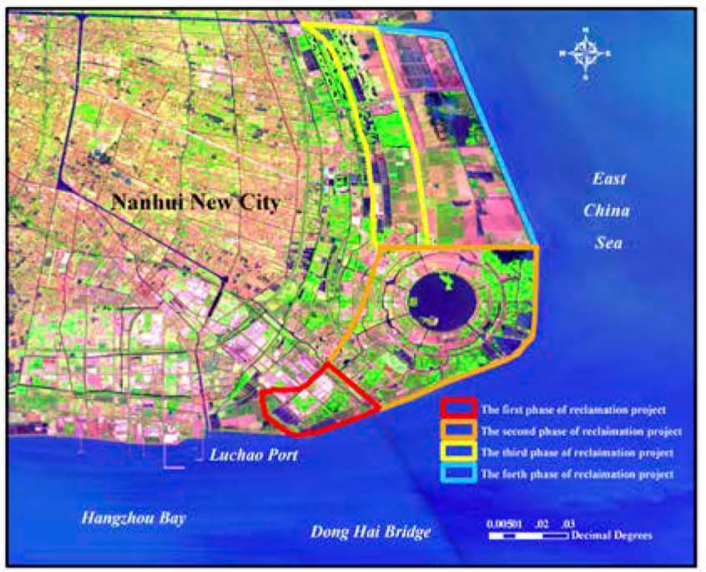

(c)

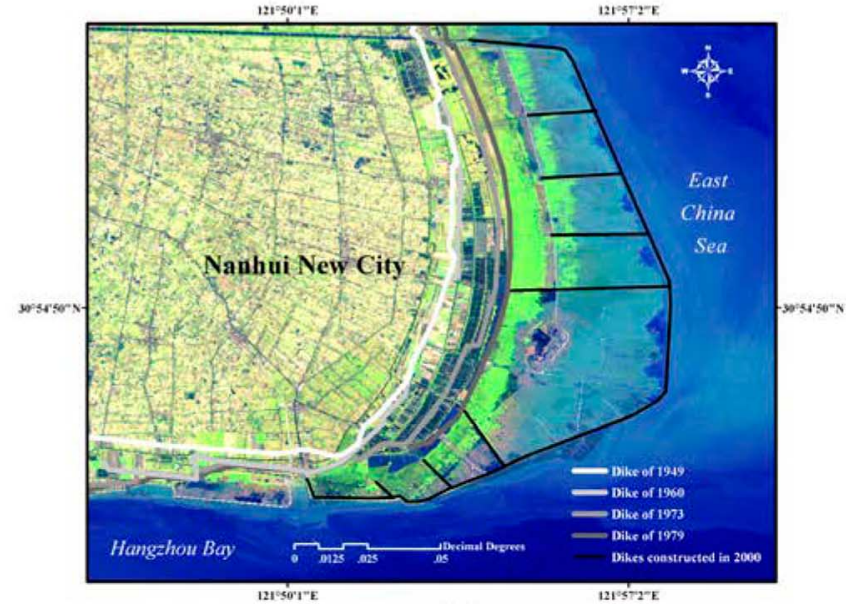

(b)

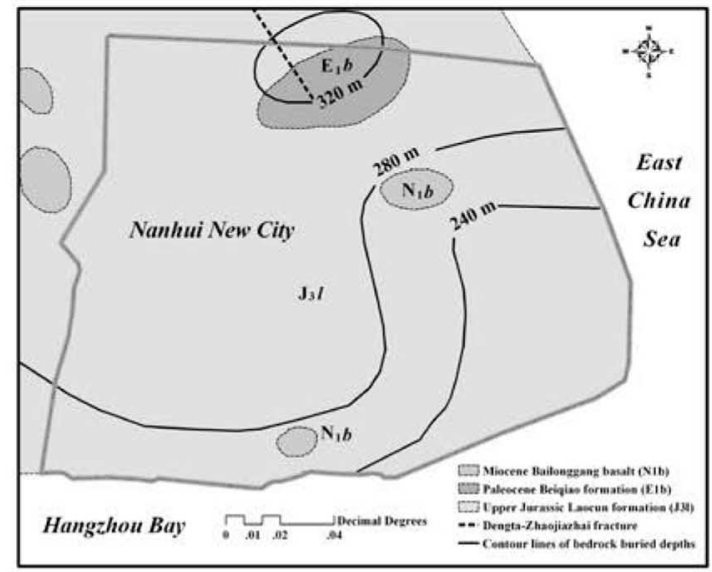

(d)

Fig. 1. Shanghai bay area. (a) Location of the Nanhui New City study area in the Shanghai metropolitan area and the distribution of the reclaimed areas from 1950 to 2020. (b) A Landsat image, collected on June 6, 2000, showing Nanhui New City and the distribution of dikes built during different time periods. (c) A Landsat image from January 1, 2013, showing Nanhui New City and the distribution and procedure of the reclamation areas. (d) A map of bedrock geology and contour lines of bedrock buried depths.

with geotechnical models that describe the expected temporal evolution of displacements during the ground consolidation phases [54], thus gathering insights into the future evolution of the settlement of reclamation areas in Nanhui New City. More precisely, we have investigated the compatibility between time-series of deformation derived from the DInSAR analysis and those expected from geotechnical analyses and laboratory centrifuge model test (CMT) experiments [54].

This paper is organized as follows. We first introduce in Section II land reclamation procedures followed by a description of the study area. SAR data and applied methodology are presented in Section III and in Section IV we provide details of the PS-SBAS cross-comparison analysis performed on the reclamation areas of Shanghai. Prediction models of the forthcoming evolution of ground displacement consolidation phase in reclaimed areas, as derived by using DInSAR measurements combined with the results of geotechnical analyses and laboratory experiments, are provided in Section V. Conclusion and further developments are provided in Section VI.

\section{Land Reclamation Procedures in Shanghai}

Land reclamation from the sea has proven to be a feasible measure for solving land scarcity in highly urbanized areas as observed in the Nanhui (formerly Lingang) New City project in Shanghai. Approximately, $133.3 \mathrm{~km}^{2}$ of land was reclaimed from 2002 to 2006, representing the initial phase of an ambitious plan developed by Shanghai authorities to reclaim $1000 \mathrm{~km}^{2}$ of new land from the mouth of the Yangtze River over a span of 20 years. Shanghai sits on the Yangtze River Delta, located on the eastern coast of China [see Fig. 1(a)]. Shanghai has the largest population in China, is surrounded by the East China Sea, the Yangtze River Estuary, and the Hangzhou Bay, and is an important commercial and financial hub for the entire country [55].

To satisfy the increasing demand for rapid industrial and urban development, Shanghai implemented large-scale land reclamation along its coastlines in the 1950s. According to statistics, the reclaimed land had already covered an area of $1014 \mathrm{~km}^{2}$ from 1953 to 2005 [56]. By 2020, a land area of 
TABLE I

Dredger Fill Type and Typical Engineering Parameter of Reclamation in Nanhui New City (Partly Referred to [58])

\begin{tabular}{|c|c|c|c|c|c|c|c|}
\hline $\begin{array}{l}\text { Alluvial } \\
\text { deposit }\end{array}$ & $\begin{array}{l}\text { Spatial } \\
\text { distribution }\end{array}$ & $\begin{array}{l}\text { Summary } \\
\text { description }\end{array}$ & $\begin{array}{l}\text { Typical } \\
\text { thickness } \\
\text { (m) }\end{array}$ & $\begin{array}{l}\text { Water } \\
\text { content } \\
(\%)\end{array}$ & $\begin{array}{l}\text { Bulk } \\
\text { density } \\
\left(\mathrm{kN} \cdot \mathrm{cm}^{-3}\right)\end{array}$ & $\begin{array}{l}\text { Void } \\
\text { ratio }\end{array}$ & $\begin{array}{l}\text { Coefficient of } \\
\text { permeability for } \\
\text { vertical flow } \\
\left(\mathrm{cm} \mathrm{a}^{-1}\right)\end{array}$ \\
\hline Type A & $\begin{array}{l}\text { Dishui Lake } \\
\text { and area east } \\
\text { of } 1973 \text { dike }\end{array}$ & $\begin{array}{l}\text { Silty soil, } \\
\text { saturated, } \\
\text { slightly dense }\end{array}$ & $>3$ & 30.7 & 18.6 & 0.86 & 2.88 \\
\hline Type B & $\begin{array}{l}\text { West Dishui } \\
\text { Lake and area } \\
\text { between } \\
1973 \\
\text { and } \\
\text { dikes }\end{array}$ & $\begin{array}{l}\text { Clay soil, } \\
\text { saturated, flow } \\
\text { plastic }\end{array}$ & $2-3$ & 38.5 & 17.6 & 1.11 & 6.43 \\
\hline
\end{tabular}

$40 \mathrm{~km}^{2}$ along the Pudong District coastline is also expected to be reclaimed.

The Nanhui New City study area is a planned industrial park development in an international shipping centre, covering an area of $300 \mathrm{~km}^{2}$, with a population of approximately 600000 inhabitants. Approximately, $45 \%$ of the land was reclaimed from intertidal wetlands. The reclaimed foundation for most areas has been in a settlement process since the project filling that was completed in 2006. Field measurements conducted at the time of reclamation completion indicated that significant ground settlement had occurred during construction of the new city [57]. The reclaimed area of Nanhui New City had been intertidal flats, where the ebb currents converge from the Yangtze River Estuary and the Hangzhou Bay, and the flood currents diverge in the opposite direction. The flats extend seaward over a maximum distance of about $20 \mathrm{~km}$ and have a very low topographic gradient. Since the levels between the Yangtze River and the Hangzhou Bay are remarkable, the sediment transport is across the flats [58], with the high flats enclosed by dikes. A Landsat image of the study area, collected on June 6, 2000, is shown in Fig. 1(b), where the distribution of the dikes constructed during different periods is very clear and effective.

It is worth repeating that ground settlement represents an inherent problem for land reclamation areas, potentially causing ground failures and infrastructure damages. Settlement can be caused by various reclamation processes, such as primary consolidation or long-term secondary compression of alluvial deposits, deposits beneath the reclamation, as well as creep within reclamation fill [59]. Reclamation settlement may occur during foundation construction and after reclamation completion. The consolidation process is typically comprised of two stages: primary consolidation and secondary compression phases. Note that the settlement due to primary consolidation is faster, and accounts for most of the total settlement (typically $70 \%$ or more). Moreover, it has been observed that the ground settlements of coastal reclaimed areas in Shanghai have been caused by over pumping groundwater and consolidation of large-area dredger fill and alluvial deposits [38].

Additional information on reclamation processes and geology of the study area are reported in the following.

\section{A. Reclamation Procedure and Dredger Fill of Nanhui New City}

The reclamation procedure of Nanhui New City is illustrated in Fig. 1(c). The first phase (enclosed with red lines) started in 1994 and was completed in 1995. From 2002 to 2004, the central area (enclosed with orange lines) was reclaimed. The northern area (enclosed with yellow lines) was reclaimed from 2003 to 2006, followed by the sector enclosed with blue lines. Sand dams, border dikes, and dredger fill methods were used to promote the siltation and rapid progress of the land formation. Reclamation procedures were performed on the eastern sector of the new city during different time periods. The areas located between the dikes built in 1973 and 1994 were reclaimed earlier than those located East of the 1994 dike which are still in the reclaiming process. According to the engineering geology characteristics of alluvial soil, hydraulic fill soil is typically composed of two types of alluvial deposits [58] (see Table I). In particular, primary consolidation of alluvial deposits in a newly reclaimed area, where the Type A alluvial deposit was used for reclamation, remains in process from 2002 until today. Field observations also indicate that primary consolidation of Type B (see Table I) alluvial deposit in areas East of the 1994 dike have not yet been completed, and remarkable residue settlement is observed.

\section{B. Bedrock Geology and Tectonics}

The bedrock found in Nanhui New City is buried at depths of $240-320 \mathrm{~m}$. The strata of the city are of upper Jurassic Laocun formation (J31) (composed of tuffaceous clastic rock, 


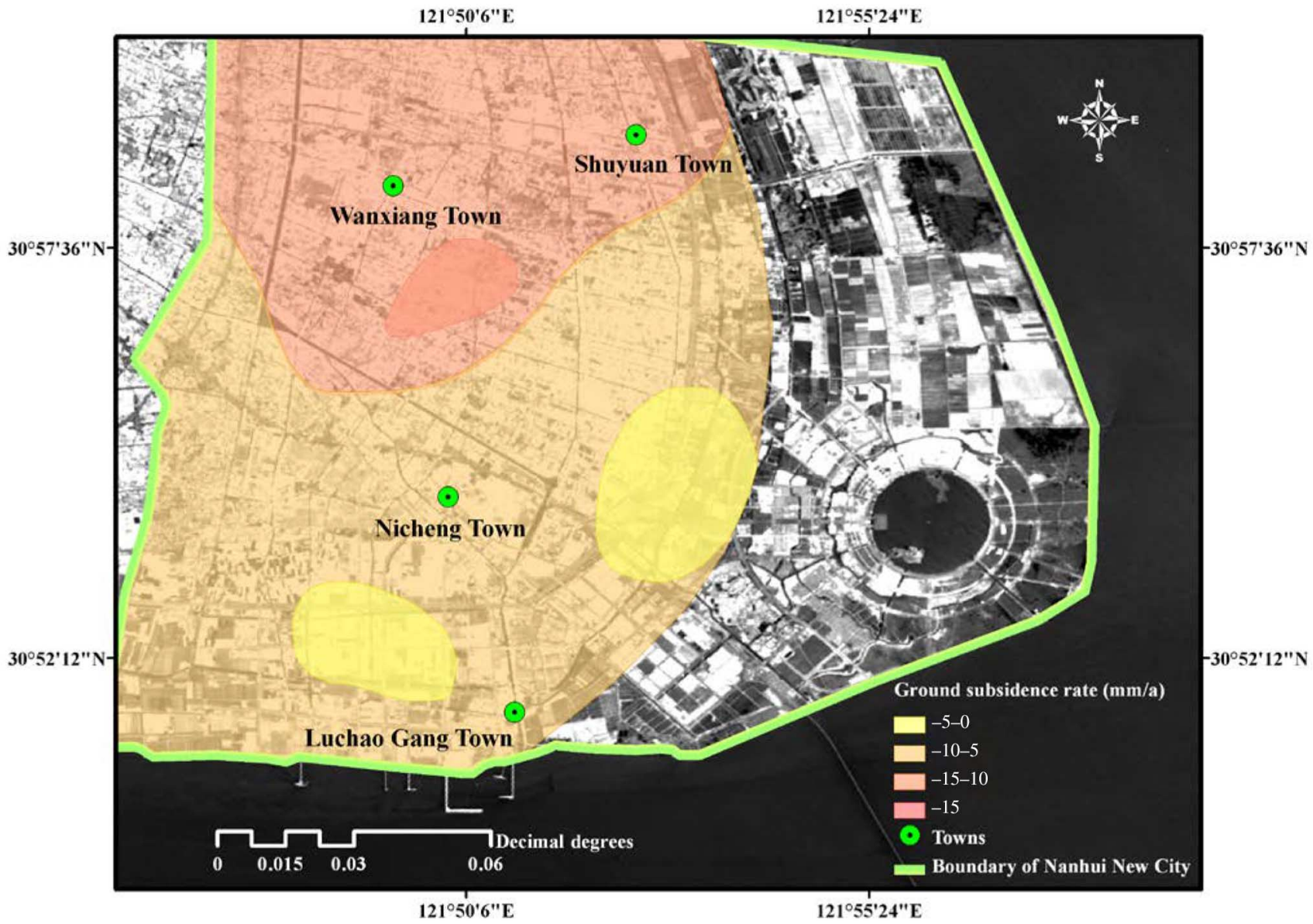

Fig. 2. Distribution of ground subsidence in Nanhui New City from 2001 to 2006 (partly referred to [62]-[64]).

tuff lava, and sandstone), Paleocene Beiqiao formation (E1b), and Miocene Bailonggang basalt (N1b) (composed of clastic and basalt rocks [see Fig. 1(d)]). This new structure, known as the Dengta-Zhaojiazhai fracture, was developed in the city and adjacent areas. The NNW-trending fracture is located North of the city with no sign of recent movement since Holocene.

\section{Engineering Geology}

Old channels pass through the new city, with the eastern sections covered by dredger fill. As a result, engineering geological conditions vary greatly across the city. The soil, ranging from 0 to $100 \mathrm{~m}$ in depth, is divided into ten engineering geological layers based on their origin, geological age, soil behavior, and physical and mechanical properties [58]-[64]. The soil found at a depth of $50 \mathrm{~m}$ below the surface of Shanghai is principally composed of clay and sand deposits. This area is typically used for underground space exploitation resulting in poor geological conditions, such as sand liquefaction and soft soil deformation.

\section{Previous Field Observed Ground Deformation}

Previous investigations indicate that ground subsidence in Nanhui New City has occurred since 1980 [64]. In East Shuyuan Town, located in the north-eastern sector of the city, cumulative settlement reached up to $75 \mathrm{~mm}$ from 1980 to 1995 with ground settlement at about $5 \mathrm{~mm} /$ year. In the West
Shuyuan Town, cumulative settlement was greater than $75 \mathrm{~mm}$, with a rate greater than $5 \mathrm{~mm} / \mathrm{year}$. In the central areas of the city, cumulative settlement reached the greatest value of $100 \mathrm{~mm}$. Throughout most of the southern area, cumulative settlement was less than $75 \mathrm{~mm}$ and ground subsidence rate was moderate. From 1996 to 2001, ground subsidence was accelerated, and a ground subsidence funnel was formed. In the area around Wanxiang Town and Shuyuan Town, the highest cumulative settlement nearly reached $200 \mathrm{~mm}$, with a ground settlement rate greater than $40 \mathrm{~mm} /$ year. In South Luchao Gang Town, cumulative settlement was greater than $75 \mathrm{~mm}$ and the annual ground settlement was up to $15 \mathrm{~mm} /$ year, as shown in Fig. 2. A sharp increase in the amount of underground water pumping caused this rapid subsidence. Generally speaking, from 2001 to 2006, both the cumulative settlement and the rate of annual ground subsidence were greater in the northern sector of the city than in the southern sector. The maximum annual ground subsidence rate was approximately $15 \mathrm{~mm} /$ year revealing a general decrease when compared with rates over the last 5 years.

\section{DAta AND Methodology}

To perform our analysis, we retrieved LOS mean displacement velocity maps as well as deformation time-series in Nanhui New City, by using both the IPTA approach [27], [65], 
TABLE II

ENViSat ASAR Acquisition Dates, Perpendicular Baselines Relative to Reference Scene, AND Time Intervals Relative to REFERENCE SCENE

\begin{tabular}{|c|c|c|c|c|}
\hline Day & Month & Year & Baseline (m) & Interval (days) \\
\hline 26 & 02 & 2007 & -175 & -315 \\
\hline 02 & 04 & 2007 & 371 & -280 \\
\hline 07 & 05 & 2007 & -198 & -245 \\
\hline 111 & 06 & 2007 & -146 & -210 \\
\hline 16 & 07 & 2007 & 31 & -175 \\
\hline 20 & 08 & 2007 & 231 & -140 \\
\hline 29 & 10 & 2007 & 64 & -70 \\
\hline 03 & 12 & 2007 & 127 & -35 \\
\hline 07 & 01 & 2008 & 0 & 0 \\
\hline 11 & 02 & 2008 & 313 & 35 \\
\hline 17 & 03 & 2008 & 102 & 70 \\
\hline 21 & 04 & 2008 & 295 & 105 \\
\hline 26 & 05 & 2008 & 7 & 140 \\
\hline 30 & 06 & 2008 & -109 & 175 \\
\hline 04 & 08 & 2008 & 30 & 210 \\
\hline 08 & 09 & 2008 & 349 & 245 \\
\hline 13 & 10 & 2008 & -221 & 280 \\
\hline 17 & 11 & 2008 & 205 & 315 \\
\hline 22 & 12 & 2008 & -149 & 350 \\
\hline 26 & 01 & 2009 & 236 & 385 \\
\hline 06 & 04 & 2009 & 198 & 455 \\
\hline 11 & 05 & 2009 & -264 & 490 \\
\hline 20 & 07 & 2009 & -88 & 560 \\
\hline 24 & 08 & 2009 & 161 & 595 \\
\hline 28 & 09 & 2009 & 166 & 630 \\
\hline 02 & 11 & 2009 & -80 & 665 \\
\hline 07 & 12 & 2009 & 245 & 700 \\
\hline 11 & 01 & 2010 & -192 & 735 \\
\hline 15 & 02 & 2010 & 226 & 770 \\
\hline 26 & 04 & 2010 & 238 & 840 \\
\hline 31 & 05 & 2010 & 51 & 875 \\
\hline
\end{tabular}

belonging to the PS group, and the SBAS algorithm [12] of the SB group. We processed a total of $N=31$ SAR acquisitions collected at VV polarization over ascending passes (Track 497 and Frame 616) by using the ENVISAT/ASAR sensor at the ordered time epochs $\mathbf{t} \equiv\left[t_{1}, t_{2}, \ldots, t_{N}\right]^{T}$, and spanning the time interval between February 2007 and May 2010 (see Table II). The available SAR images were independently processed through the IPTA and the SBAS DInSAR processing chains. A common master SAR image, namely the one collected on January 07, 2008, was selected for both algorithms and taken as a reference. From the available SAR images, two different sets of differential SAR interferograms used by the two distinctive processing chains were initially computed. In particular, within the PS/IPTA processing chain, the images were paired to identify a sequence of, say $M^{(I P T A)}$, common-master interferograms (i.e., in our case $M^{(I P T A)}=$ 30 ), and were evaluated with respect to the selected master acquisition. Conversely, for the SBAS case, an appropriate set of $M^{(S B A S)}=78$ SB SAR data pairs (see Table III) was identified by imposing constraints on the maximum spatial and temporal (baseline) separation between orbits, which have been set in our analysis to $400 \mathrm{~m}$ and 1000 days, respectively.

All the generated PS-IPTA and SBAS-DInSAR interferograms were flattened using an $80 \times 80 \mathrm{~m}$ Shuttle Radar Topography Mission (SRTM) DEM of the Shanghai area. Precise orbital information was also provided to us by the University of Delft. In regard to the SBAS case, to reduce the amount of processed data and to mitigate noise effects, a complex multilook operation [66] (with ten looks in the azimuth direction and two looks in the range direction) was independently performed on each interferogram. A space/time noise-filtering procedure recently proposed in [67], and new solutions for phase unwrapping operations [68], [69], representing further development of the SBAS processing chain, were also applied.

The diagram blocks of the IPTA and SBAS processing chains are shown in Fig. 3(a) and (b), respectively. For detailed descriptions of both algorithms, the reader is referred to literature [9], [12], [29], [65], [70]. 
TABLE III

SMALL BASELINE INTERFEROGRAMS USED Within THE SBAS-DINSAR PRocessing CHAIN

\begin{tabular}{|c|c|c|}
\hline Interferometric pair & Perpendicular baseline $(\mathrm{m})$ & Interval (days) \\
\hline $26022007 \mathrm{ENV} 11062007 \mathrm{FNV}$ & -28.0754 & 105 \\
\hline 26022007 TENV_07052007ENV & 23.1413 & 70 \\
\hline $02042007 E N V_{-} 16072007 \mathrm{ENV}$ & 341.752 & 105 \\
\hline $070520007 E N N^{-11062007 E N V}$ & -51.2122 & 35 \\
\hline 07052000TENV_16072007ENV & -229.489 & 70 \\
\hline $07052007 \mathrm{ENV} 291021007 \mathrm{ENV}$ & -261.849 & 175 \\
\hline $07052007 \mathrm{NV} 031221007 \mathrm{NV}$ & -326.48 & 210 \\
\hline $11062007 \mathrm{ENV} 116072007 \mathrm{ENV}$ & -178.677 & 35 \\
\hline $11062007 \mathrm{TNV} 200020107 \mathrm{NV}$ & -378.739 & 70 \\
\hline $11062007 \mathrm{ENV}^{-} 03122007 \mathrm{ENV}$ & -275.264 & 175 \\
\hline $16072007 \mathrm{ENV} 29102007 \mathrm{ENV}$ & $-31: 9478$ & 105 \\
\hline $16072007 \mathrm{ENV} 07012008 \mathrm{ENV}$ & 33.1019 & 175 \\
\hline $16072007 \mathrm{ENV} 20082007 \mathrm{ENV}$ & -200.066 & 35 \\
\hline $20082007 \mathrm{NVV}^{-} 03122007 \mathrm{ENV}$ & 103.512 & 105 \\
\hline $20082007 E N V^{-29102007 E N V ~}$ & 168.133 & 70 \\
\hline 29102007 TEN $03122007 E N V$ & -64.6244 & 35 \\
\hline $2910200 \pi$ TEV_17032008ENV & -38.6923 & 140 \\
\hline $29102007 E N V$ 07012008ENV & 65.0502 & 70 \\
\hline 29102007 ENV 260521008ENV & 56.8928 & 210 \\
\hline $29102007 \mathrm{ENV} 110221008 \mathrm{ENV}$ & -251.392 & 105 \\
\hline $29102007 \mathrm{NVV} 21042010 \mathrm{RENV}$ & -233.504 & 175 \\
\hline $03122007 \mathrm{ENV} 17032008 \mathrm{ENV}$ & 25,9323 & 105 \\
\hline $03122007 \mathrm{ENV} 11022008 \mathrm{ENV}$ & -186.775 & 70 \\
\hline $03122007 \mathrm{ENV} 07012008 \mathrm{ENV}$ & 129.672 & 35 \\
\hline $03122007 \mathrm{ENV}^{2} 21042008 \mathrm{ENV}$ & -168.887 & 140 \\
\hline $03122007 \mathrm{ENV}^{-26052008 \mathrm{ENV}}$ & 121.508 & 175 \\
\hline $07012008 \mathrm{ENV} 26052008 \mathrm{ENV}$ & -8.15075 & 140 \\
\hline $07012008 E N V+11022008 E N V$ & -316.439 & 35 \\
\hline $07012008 \mathrm{ENV} 17032008 \mathrm{ENV}$ & -103.745 & 70 \\
\hline $07012008 \mathrm{ENV} 21042008 \mathrm{ENV}$ & -298.549 & 105 \\
\hline 110221008ENV_210421108ENV & 17.8808 & 70 \\
\hline $11022008 \mathrm{ENV} 17032108 \mathrm{ENV}$ & 212.73 & 35 \\
\hline $11022008 \mathrm{ENV} 260521008 \mathrm{NV}$ & 36.277 & 105 \\
\hline 170320081ENV_210421008ENV & -194.818 & 35 \\
\hline $17032008 E N V \_26052008 \mathrm{ENV}$ & 95.5791 & 70 \\
\hline $21042008 \mathrm{ENV} 26052008 \mathrm{ENV}$ & 290,396 & 35 \\
\hline $26052008 \mathrm{ENV} 04082008 \mathrm{ENV}$ & -23.5737 & 70 \\
\hline $260520008 \mathrm{ENV} 30062008 \mathrm{ENV}$ & 115.503 & 35 \\
\hline $30062008 \mathrm{ENV}^{-} 04082008 \mathrm{ENV}$ & -139.068 & 35 \\
\hline $30062008 \mathrm{ENV} 17112008 \mathrm{ENV}$ & -314.85 & 140 \\
\hline $30062008 E N V_{-} 22122008 \mathrm{ENV}$ & 41.1568 & 175 \\
\hline $30062008 \mathrm{ENV} 26012009 \mathrm{ENV}$ & -345.928 & 210 \\
\hline $04082008 \mathrm{ENV} 22122008 \mathrm{ENV}$ & 180.24 & 140 \\
\hline $04082008 E N V \_17112008 E N V$ & -175.774 & 105 \\
\hline $04082008 \mathrm{ENV} 131021008 \mathrm{ENV}$ & 252.465 & 70 \\
\hline 04082008ENV_080920108ENV & -319.792 & 35 \\
\hline $04082008 \mathrm{TNV} 260120109 \mathrm{KNV}$ & -206.83 & 175 \\
\hline $08092008 E N V_{-} 17112008 \mathrm{ENV}$ & 144.105 & 70 \\
\hline $08092008 \mathrm{ENV} 26012009 \mathrm{ENV}$ & 112.933 & 140 \\
\hline $08092008 \mathrm{ENV} 06042009 \mathrm{ENV}$ & 149.682 & 210 \\
\hline $131020008 \mathrm{ENV} 22122008 \mathrm{ENV}$ & -72.2366 & 70 \\
\hline $13102008 \mathrm{ENV} 11052009 \mathrm{ENV}$ & -128.33 & 140 \\
\hline 17112008ENV_22122008ENV & 42.403 & 210 \\
\hline $17112008 \mathrm{ENV}^{-06042009 \mathrm{ENV}}$ & 356.022 & 35 \\
\hline $17112008 \mathrm{ENV} 26012009 \mathrm{ENV}$ & 5.5783 & 140 \\
\hline $22122008 \mathrm{ENV} 11052009 \mathrm{ENV}$ & -31.1421 & 70 \\
\hline $22122008 \mathrm{ENV} 06042109 \mathrm{ENV}$ & 299.734 & 105 \\
\hline $22122008 \mathrm{NV} 26012009 \mathrm{NV}$ & 114.633 & 140 \\
\hline $260120095 \mathrm{NV} 06042109 \mathrm{ENV}$ & $-350.42 \mathrm{~s}$ & 105 \\
\hline $11052009 E N V=20072009 \mathrm{ENV}$ & $-56,2932$ & 70 \\
\hline $20072009 \mathrm{ENV} 24082009 \mathrm{ENV}$ & -387.075 & 35 \\
\hline 20072009ENV $02112009 \mathrm{ENV}$ & 36.7263 & 70 \\
\hline 20072009ENV 28092009ENV & 330.884 & 35 \\
\hline $24082009 \mathrm{ENV}^{2} 20092009 \mathrm{ENV}$ & 170.922 & 70 \\
\hline 24082009ENV_07122009ENV & -294.135 & 35 \\
\hline 24082009ENV_02112009ENV & -3.61265 & 140 \\
\hline $28092009 \mathrm{ENV} 11012010 \mathrm{ENV}$ & -176.546 & 70 \\
\hline $28092009 \mathrm{ENV} 07122009 \mathrm{ENV}$ & -249.527 & 35 \\
\hline 28092009ENV_02112109ENV & -7.16543 & 105 \\
\hline 02112009ENV_11012010ENV & -256.744 & 70 \\
\hline 021120091ENV_071220109ENV & $-7,22126$ & 35 \\
\hline $021120095 \mathrm{NV} 15022010 \mathrm{ENV}$ & $-84,8691$ & 105 \\
\hline $02112009 \mathrm{ENV} 26042010 \mathrm{ENV}$ & 242,422 & 70 \\
\hline $07122009 \mathrm{ENV} 31052010 \mathrm{ENV}$ & 361,681 & 105 \\
\hline $07122009 \mathrm{ENV} 15022010 \mathrm{ENV}$ & -77.6476 & 70 \\
\hline I5022010ENV_31052010ENV & 249.642 & 35 \\
\hline 15022010ENV 26042010ENV & 112.06 & 70 \\
\hline $26042010 \mathrm{ENV}^{-} 31052010 \mathrm{ENV}$ & -327.257 & 35 \\
\hline
\end{tabular}

To have a common grid for the subsequent analyses, all IPTA and SBAS DInSAR products were subsequently geocoded to the SRTM DEM of the area. Note also that both DInSAR products provide relative measurements, computed with respect to a common reference point that is assumed stable. In our case, this point was identified in the zone just outside the reclamation platforms with Type A and Type B soil characteristics (see Fig. 4), where the reclamation process is nearly concluded, and in the vicinity of the levelling benchmark as identified with a red star in Fig. 4(a). The area under consideration is almost stable, as confirmed by the average ground settlement rate of $0.4 \mathrm{~mm} /$ year, as recorded at the levelling benchmark from May 2009 to May 2010 [see Fig. 4(b)].

Finally, as a result of the IPTA and SBAS processing steps, we retrieved two independent sets of (geocoded) DInSAR displacement time-series, namely $\left\{d^{(P S)}(e, n, \tau) \forall P \equiv(e, n) \in S^{(I P T A)}, \tau \in \mathbf{t}\right\} \quad$ and $\left\{d^{(S B)}(e, n, \tau) \forall P \equiv(e, n) \in S^{(S B)}, \tau \in \mathbf{t}\right\}, \quad$ computed in correspondence to highly coherent PS and DS target locations, $S^{(I P T A)}$ and $S^{(S B A S)}$, into the geocoded domain, respectively.

For the IPTA case, we retrieved a network of about $20 \mathrm{~K}$ coherent PS targets (about $6 \mathrm{~K}$ of which were located in the reclaimed platforms), obtained by imposing a threshold of $1.1 \mathrm{rad}$ for the standard deviation value between the displacement time-series and a 2-D regression-model in the temporal/perpendicular baseline plane (see [27] for more details). Conversely, for the SBAS case, a network composed by almost $35 \mathrm{~K}$ DS targets was identified by using a temporal coherence [71] threshold of 0.7 .

At this stage, the two sets of DInSAR products were jointly analyzed to isolate a group of very coherent and phasepreserving scatterers in the reclaimed areas of Shanghai. These were used to investigate the validity of a geotechnical model derived from laboratory tests, to then predict the future evolution of displacement in reclaimed platforms.

The description of the cross-comparison strategy we adopted to jointly investigate PS-IPTA and SBAS deformation products, as well as the results of such an analysis, is reported in Section IV. There is a twofold justification for performing a cross-comparison analysis between advanced IPTA and SBAS DInSAR techniques over the investigated ocean-reclaimed lands of Shanghai. First, it allows for isolation of a group of very stable DS and PS targets with high accuracy deformation measurements. Second, but not less important, the proposed PS-SBAS cross-comparison analysis is profitable for assessing the retrieval accuracy of DInSAR-based deformation time-series in the presence of SAR scenes characterized by rapidly time-varying geometrical and electromagnetic conditions, which lead to significant temporal decorrelation artifacts in the generated interferograms. As a matter of fact, very few cross-comparison analyses have been conducted to date between the two families of PS and SB techniques [35], [50], [51], [53], [72], [73]. Those that have been conducted were at regional scale over urbanized and/or from moderate to high coherence levels. To our knowledge, no comparison analysis has singularly focused on regions subject to severe and long-lasting reclamation processes.

\section{JOINT ANALYSIS OF PS-IPTA AND SBAS RESUlTS}

We applied the PS-IPTA and SBAS processing chains to the available set of SAR images, and as previously stated, 


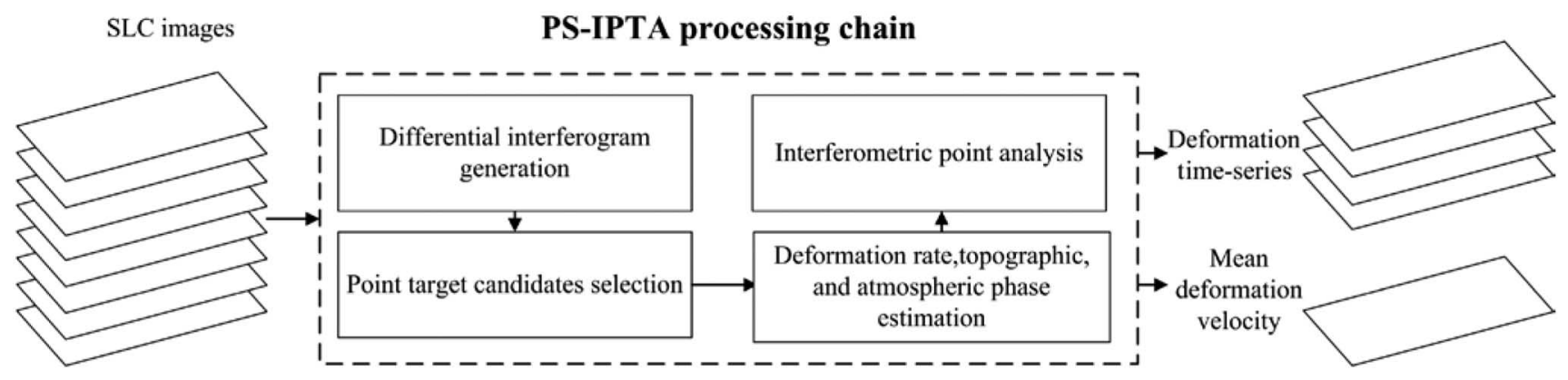

(a)

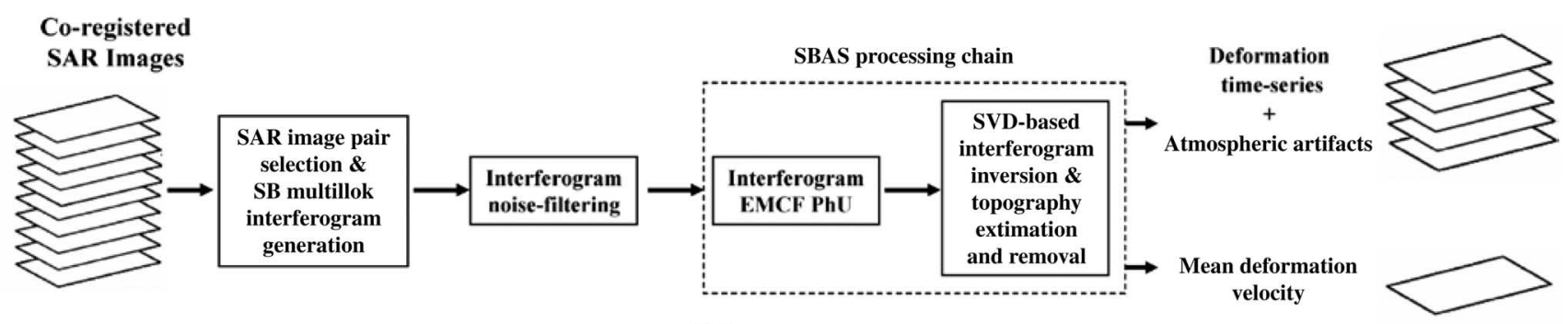

(b)

Fig. 3. Diagram block of the implemented (a) PS-IPTA and (b) SBAS processing chains.

we preliminarily identified two sets of coherent PS and DS targets, namely $S^{(I P T A)}$ and $S^{(S B A S)}$, respectively. We show, in Fig. 5(I) and 5(II), the geocoded mean deformation velocity maps of Nanhui New City, as retrieved by applying the IPTA and SBAS processing chains, respectively. The maps only portray the coherent PS/DS targets that correspond to the sets of $S^{(I P T A)}$ and $S^{(S B A S)}$ points, whereas incoherent targets have been discarded. The maps are superimposed on the Landsat image of the area taken on June 6, 2000. It is evident that both techniques identify the main deformation features of the Nanhui New City and are, at most, consistent with each other. As expected, PS targets are primarily detected on man-made infrastructures and buildings. Alternatively, only a few targets are present in other areas such as North and East of Dishui Lake, where land reclamation has not been completed and thus remains rural. The DS targets are clearly detectable in these rural areas. However, SBAS suffers from temporal decorrelation noise effects in lands that were still reclaiming during the time the SAR images were taken. IPTA and SBAS-DInSAR results, in correspondence to homologous PS/DS target locations, show a maximum (subsidence) deformation rate of approximately $5 \mathrm{~cm} /$ year and indicate a stable pattern in the central and western sections of the new city. The reclaimed projects were completed in these areas before 1950 and sediments were consolidated over a period of 60 years, with an average ground settlement rate less than $7 \mathrm{~mm} / \mathrm{year}$ (green spots). Localized areas with moderate to high deformation rates (higher than $2 \mathrm{~cm} /$ year) are clearly identifiable in both the northern and southern districts of the urban area (see orange spots). We observe an overall good agreement between the two deformation maps as shown in Fig. 5(I) and (II), except for the aforementioned areas in North Dishui Lake and just in the near proximity of coastline where the difference between deformation values is larger. Note that detection of coastline ground deformation in our case is very critical, due to its continuous changes during the period of observation, thus affecting the accuracy of DInSAR measurements. Nonetheless, SBASDInSAR measurements corresponding to the DS targets that encompass the entire coastline are generally in agreement with in situ levelling data (see those reported in [74]).

We start the cross-comparison analysis by first focusing on the difference in the mean LOS velocities as estimated by the PS-IPTA and SBAS techniques. To perform such a comparison, coherent DS and PS targets should fall inside the same cell of the geocoded grid. However, this is not generally guaranteed. To overcome this limitation, we applied the following strategy. ${ }^{1}$ We first identified each PS target of $S^{(I P T A)}$, and for the given $p$ th generic PS point, we compared the IPTA displacement time-series, say $d^{(P S)}\left(e_{p}, n_{p}, \tau\right) \quad \forall \tau \in \mathbf{t}$, to the (average) displacement time-series of the $Q$ DS targets that are located in near proximity of the considered $p$ th PS target. More precisely, we concentrated on an area of about $200 \times 200 \mathrm{~m}$ centred on the position of the $p$ th PS target. We then identified the $Q$ displacement time-series, namely $d^{(S B)}\left(e_{q}, n_{q}, \tau\right) \quad \forall q=1, \ldots, Q \forall \tau \in \mathbf{t}$, which corresponds to its neighboring DS targets. We concluded by computing a (weighted) average DS-SBAS displacement time-series as follows:

$$
\bar{d}^{(S B)}\left(e_{p}, n_{p}, \tau\right)=\frac{\sum_{q=1}^{Q} \Gamma\left(Q_{q}\right) d^{(S B)}\left(e_{q}, n_{q}, \tau\right)}{\sum_{q=1}^{Q} \Gamma\left(Q_{q}\right)} \quad \forall \tau \in \mathbf{t}
$$

\footnotetext{
${ }^{1}$ Actually, here we are more interested in the spatial low-pass (LP) displacement signal components, associated more to ground movement and less to relative motion of man-made structures w.r.t. the ground.
} 


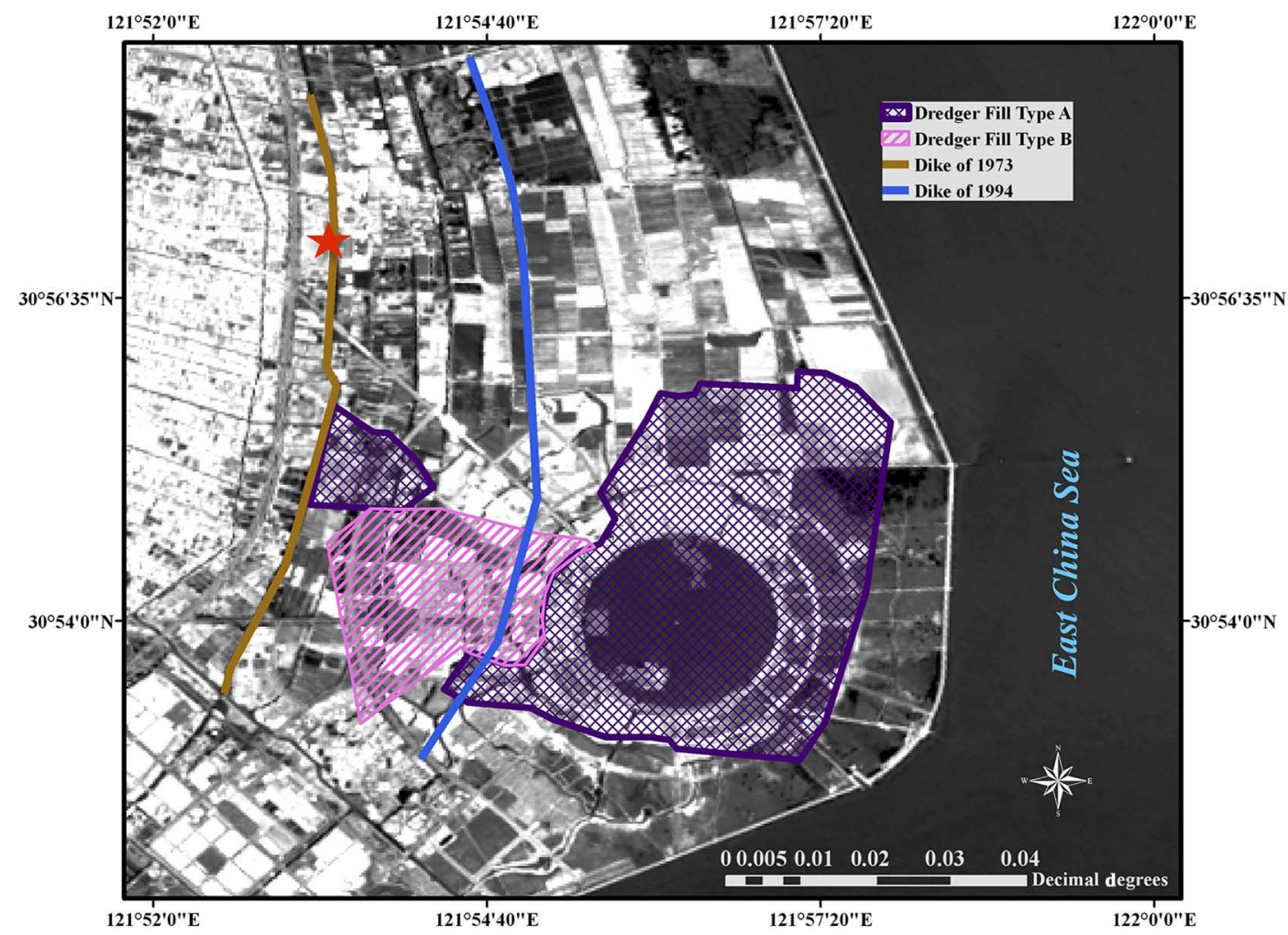

(a)

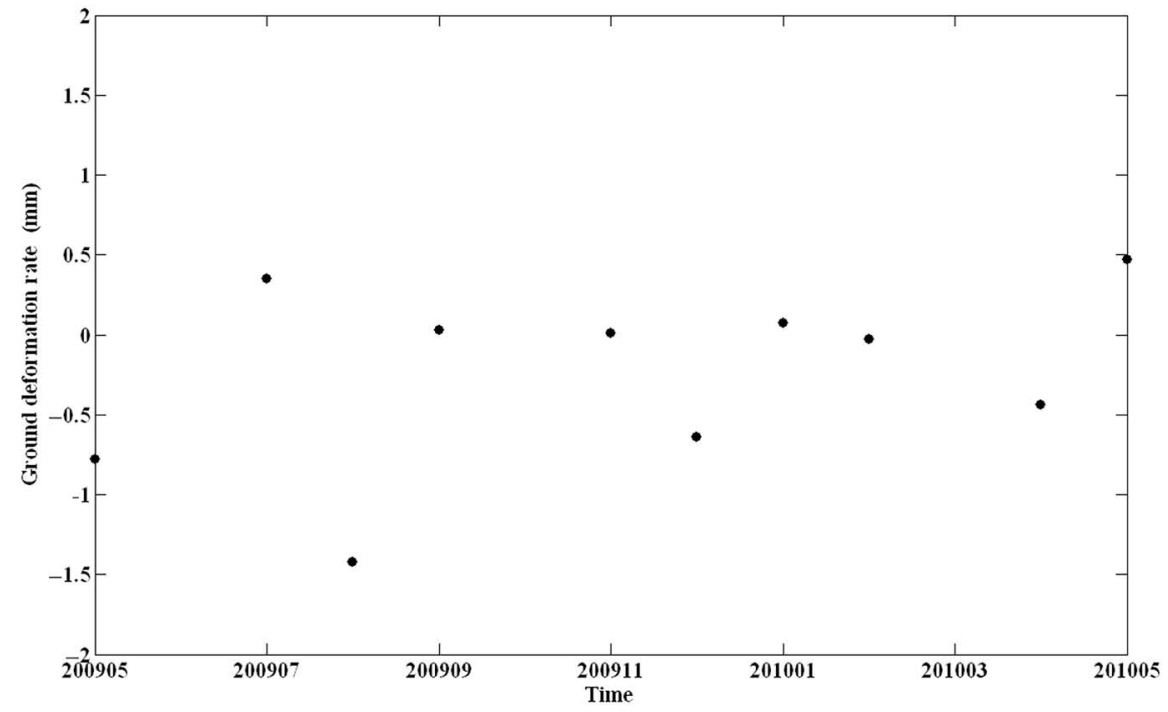

(b)

Fig. 4. Nanhui New City: (a) Distribution of alluvial deposits within Type A (purple) and Type B (pink) dredger fill areas. (b) Vertical displacement time-series of the ground, as measured from May 2009 to May 2010 in correspondence with the levelling station identified with the red star in (a).

where the weights represent the SBAS temporal coherence values that correspond to the $Q$ DS targets. At first, we computed the (average) DS mean displacement rate, namely $\bar{V}^{(S B)}\left(e_{p}, n_{p}\right)$, via a simple one dimensional (1-D) linear regression of the (average) displacement time-series $\bar{d}^{(S B)}\left(e_{p}, n_{p}, t\right)$ as a function of time $\mathbf{t}$.

We started our analysis by comparing the IPTA $V^{(P S)}\left(e_{p}, n_{p}\right)$ and the SBAS $\bar{V}^{(S B)}\left(e_{p}, n_{p}\right)$ velocities. The good agreement between these two different DInSARbased measurements can easily be (and synthetically) checked by plotting IPTA velocities versus SBAS velocities, as shown in Fig. 6(a). Note that points located away from the line of equation $V^{(P S)}=\bar{V}^{(S B)}$ correspond to critical areas, where reclaiming procedures were still active from 2007 to 2010, most of which were located near the coastline. However, discrepancies larger than $3 \mathrm{~mm} / \mathrm{year}$ represent only $10 \%$ of the entire population of common DS/PS targets. However, this percentage diminishes to about $1 \%$ if we consider velocity differences larger than $10 \mathrm{~mm} / \mathrm{year}$. We also portray, in Fig. 6(b), the histogram of the SBAS/IPTA velocity differences 

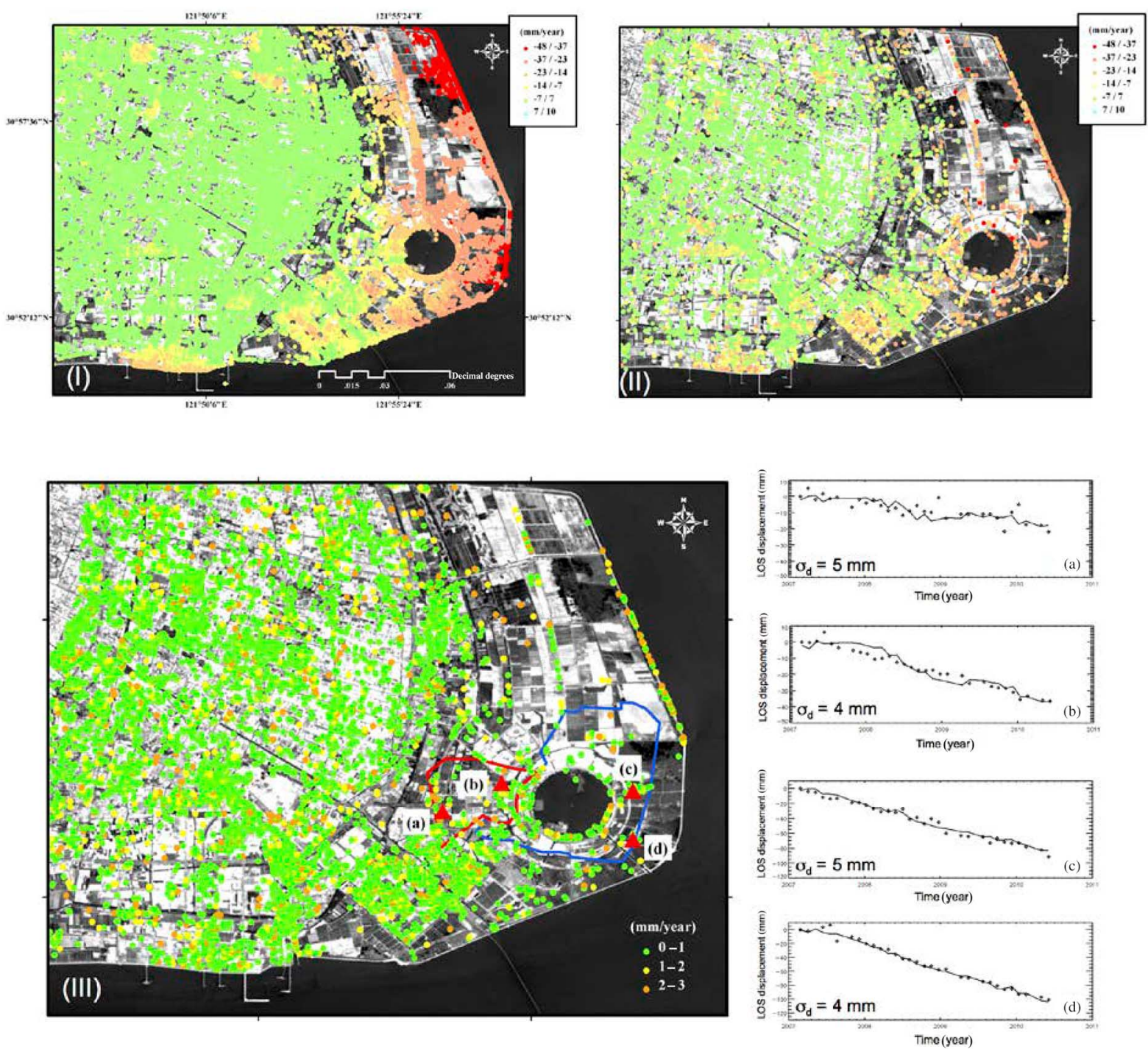

Fig. 5. SBAS/PS-IPTA intercomparison analysis results: (I) SBAS LOS mean deformation velocity map, superimposed on a Landsat image of the area and portraying pixels with temporal coherence values greater than 0.7 , only. (II) IPTA LOS mean deformation velocity map of the study area; only "good" PS targets are shown. (III) Map of the LOS velocity difference between IPTA and SBAS DInSAR products. To highlight the velocity value differences for most pixels only the DS/PS targets with IPTA/SBAS velocity difference values less than $3 \mathrm{~mm} /$ year are shown here. The standard deviation values of the IPTA/SBAS difference timeseries are less than $1 \mathrm{~cm}$. Plots (a)-(d) show the comparison between SBAS (continuous lines) and IPTA (black stars) deformation time-series in correspondence to pixels labelled as (a)-(d) in (III). The standard deviation values of these time-series differences are also shown for each selected point.

computed in correspondence to the homologous DS/PS targets. Numerically, the average displacement difference between IPTA and SBAS measurements in common pixels, is $-1.5 \mathrm{~mm} /$ year. This is equal to $0.9 \mathrm{~mm} /$ year if we exclusively focus on the two Type A/Type B reclaimed regions, which are highlighted by red and blue contour lines, respectively, in Fig. 5(III).

It is worth noting that the PS displacement signals, especially in correspondence to buildings and/or man-made structures, also take into account possible differential movements between these structures and the ground, whereas SBAS signals (computed on multilook interferograms) are typically sensitive to the ground deformation.

In addition to conducting comparisons of the mean deformation velocities, we also directly compared the difference of the estimated time-series, i.e., $d^{(P S)}\left(e_{p}, n_{p}, \tau\right)-$ $\bar{d}^{(S B)}\left(e_{p}, n_{p}, \tau\right) \quad p=1, \ldots, P \quad \forall \tau \in \mathbf{t}$. We then calculated the standard deviation of the difference, namely $\sigma_{D}$, which can be considered as a further quality factor for the correct identification of a set of very coherent DS/PS targets (to be used for the following analyses). The histogram of the standard deviation of the difference between the PS-IPTA and SBAS deformation time-series is shown in Fig. 7. As a result, the IPTA/SBAS standard deviation values for all of Nanhui New City and in the Type A/Type B regions measured 6 and $5 \mathrm{~mm}$, respectively. Note that the measured values of the (average) mean displacement velocity and displacement time-series are in good agreement with those expected by previous intercomparison investigations, and demonstrate that the reclaimed regions (after a certain period following the end of reclamation, and during consolidation phases) can be accurately monitored through DInSAR-based analyses. To give even greater evidence that most of the areas under investigation exhibit a good fit between PS and DS displacements, we 
observe that almost $87 \%$ of the whole set of PS targets retrieved using the IPTA processing are characterized by a IPTA/SBAS standard deviation difference less than $10 \mathrm{~mm}$ and a mean velocity difference less than $3 \mathrm{~mm} /$ year. Moreover, this percentage is reduced to $78 \%$ if calculated over Type A/Type B regions. The map of the velocity difference computed in correspondence to this subset of points is shown in Fig. 5(III). It is evident that most of the points have a velocity difference less than $1 \mathrm{~mm} /$ year (green points). Spotted areas with differences of 2-3 $\mathrm{mm} /$ year are also present over the southern sector of coastline. PS/DS targets exhibiting larger velocity differences and standard deviation values are not shown in Fig. 5(III). Furthermore, four points located in the two Types A and B dredger fill platforms, and labelled as (a), (b), (c), and (d) in Fig. 5, were selected. In correspondence to these four pixels, Fig. 5 shows both the comparison between IPTA and SBAS displacement time-series and the values of the measured IPTA/SBAS standard deviation. Note that PS time-series have more fluctuations than the SBAS ones. These differentiations are due to the presence of localized displacement components in the PS time-series relevant to the differential movements of man-made structures with respect to the ground, and the fact that SBAS time-series are computed at a multilook resolution scale.

Finally, as a result of this cross-comparison analysis, we identified a set of very coherent points in both Types A and B dredger fill platforms, characterized by a (measured) IPTA/SBAS velocity difference of less than $1 \mathrm{~mm} / \mathrm{year}$ and an IPTA/SBAS standard deviation difference less than $5 \mathrm{~mm}$. Based on these selected points, some additional tests, aimed at analyzing and/or forecasting the temporal evolution of displacement in next years, were performed.

Our results demonstrate that, even in a critical region, such as the one represented by a reclaimed land, DInSAR accuracy is mostly preserved, and is in good agreement with that provided by previous studies [54], [57], [64], [76], [77]. The principal results are summarized in Table IV.

As a final remark, we observe that DInSAR measurements only represent the component of the deformation along the sensor-to-target LOS direction [1]. However, as confirmed by previous studies [54] in reclaimed areas, it is reasonable to assume that most of the deformation is vertical. Accordingly, to perform the following analyses, we imposed that the deformation is typically vertical, and thus converted all IPTA and SBAS-derived LOS measurements in vertical movements using the following relation:

$$
d i s p_{\text {vert }} \cong d i s p_{L O S} / \cos \vartheta
$$

where disp $_{\text {vert }}$ and $d i s p_{L O S}$ are the vertical and LOS deformation components, respectively, and $\vartheta$ is the sensor side-looking angle.

\section{Geotechnical Analysis for Settlement MEASUREMENT}

In this section, we present one model describing the temporal evolution of deformation in reclaimed areas, as derived

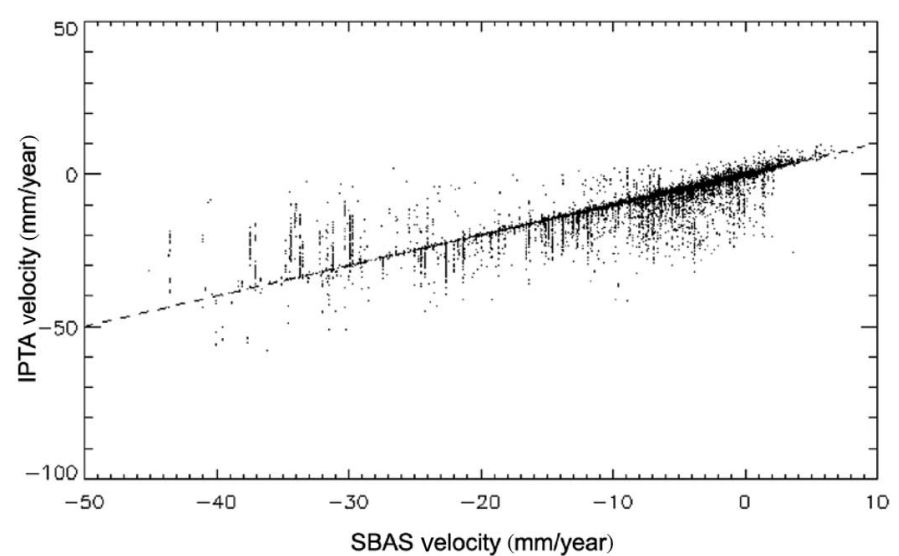

(a)

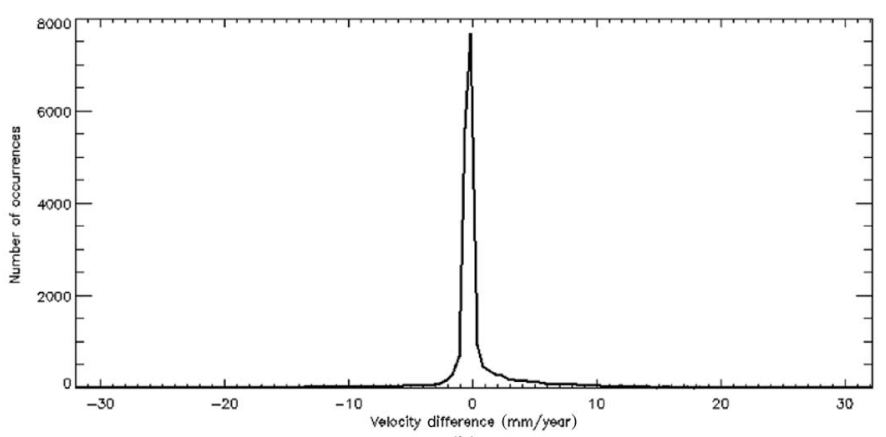

(b)

Fig. 6. SBAS/PS-IPTA cross-comparison analysis results. (a) SBAS velocity vs. PS-IPTA velocity scatter plot (represented by black dots). It is important to note that $87 \%$ of the DS/PS targets lie in close proximity to the straight line (dashed line) defined by the equation $V^{(P S)}=\bar{V}^{(S B)}$, which is characterized by an LOS velocity difference less than $3 \mathrm{~mm} /$ year. (b) Histogram of the LOS velocity difference between IPTA and SBAS.

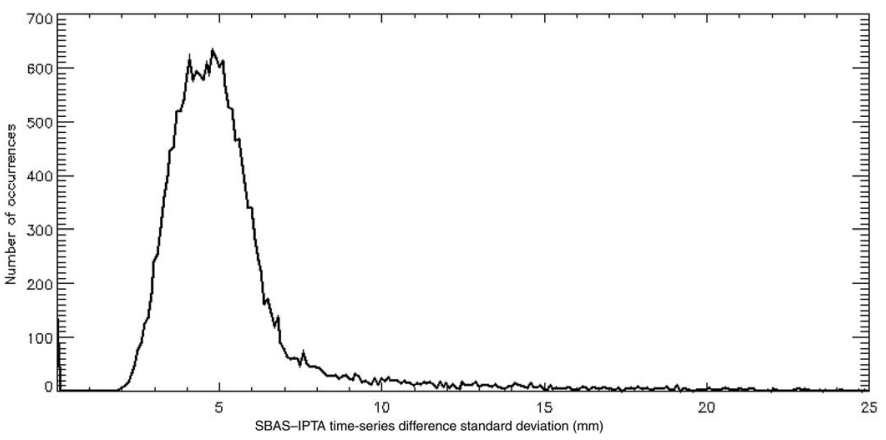

Fig. 7. Results of the SBAS/IPTA cross-comparison analysis. Histogram of the SBAS/IPTA LOS difference displacement time-series standard deviation.

from geotechnical estimates of the ground displacement signals affecting reclamation areas.

Settlement due to reclamation will continue over the lifetime of the reclamation facility, and can be distinguished in a primary consolidation phase and in a secondary compression stage of the alluvial deposit creep of the reclamation fill [54]. The majority of settlement due to primary consolidation takes place immediately after the completion of the reclamation process, and may continue for dozens of years. To generate a complete time-settlement curve for reclamation fill and make 
TABLE IV

2007-2010 Ground Subsidence Derived by Our DinSAR-BASEd Investigation ANd From Previous Studies

\begin{tabular}{|c|c|c|c|}
\hline $\begin{array}{l}\text { Ground subsidence monitoring } \\
\text { methods }\end{array}$ & Annual rate of subside & year) & \\
\hline \multirow{3}{*}{ IPTA } & Type A of dredger fill & Average value & 22 \\
\hline & Type B of dredger fill & Average value & 9 \\
\hline & Dredger fill & Average value & 18 \\
\hline \multirow{3}{*}{ SBAS } & Type A of dredger fill & Average value & 24 \\
\hline & Type B of dredger fill & Average value & 8 \\
\hline & Dredger fill & Average value & 20 \\
\hline Centrifuge modelling test ${ }^{1}[54]$ & \multicolumn{3}{|l|}{19} \\
\hline Step benchmarks of littoral area $^{2}[58]$ & \multicolumn{3}{|l|}{13} \\
\hline $\begin{array}{l}\text { Levelling and GPS benchmarks of } \\
\text { Shanghai }^{2}[54],[75]\end{array}$ & \multicolumn{3}{|l|}{12} \\
\hline $\begin{array}{l}\text { Levelling benchmarks of reclamation } \\
\text { area in Nanhui city [74] }\end{array}$ & Average value & 16 & \\
\hline
\end{tabular}

${ }^{1}$ Note that these are modelled deformation values, not measured ones.

${ }^{2}$ Note that these measurements are not freely available for comparison purposes, and the exact locations of them is unknown.

long-term predictions of the magnitude of consolidation settlement, geotechnical models have been established by using settlement monitoring data and geotechnical tests [54], [59].

A geotechnical centrifuge model has been used for conducting some tests on geotechnical problems, with the aim to assess stability and capacity of building foundations. Geotechnical materials (e.g., soils) have nonlinear mechanical properties, which depend on stress state and stress path. The basic principle of centrifuge is to recreate the stress conditions that would exist in a full-scale construction (prototype), using a model on a reduced scale. This is obtained by subjecting the model components to an enhanced body force, which is provided by an increased gravitational acceleration. The one-to-one scaling of stress enhances the similarity of geotechnical models and makes it possible to obtain accurate data to help solve complex geotechnical problems. Centrifuge model testing provides data to improve our understanding of basic mechanisms of deformation, and allows us to obtain experimental data for verification of numerical models [76].
Centrifuge modeling has the ability to reproduce the same stress levels as the prototype in a small-scale model. This CMT is a useful tool for the simulation and prediction of long-term subsidence [77], [78]. Of particular interest, due to the alluvial soil characteristics of Shanghai, is the model settlement derived by Yang et al. [54] from laboratory centrifuge tests. This laboratory model permits us to analyze ground settlement caused by self-weight consolidation [79]. In this model, the relationship between self-weight consolidation settlement and time $t$ is considered as follows:

$$
s_{C M T}(t)=S_{m} \frac{(t-\delta)^{\lambda}}{k^{\lambda}+(t-\delta)^{\lambda}}
$$

where $\mathrm{t}$ is the consolidation time (including both primary consolidation and secondary compression phases). In (3), $S_{m}$ represents the asymptotic (total) settlement assumed at infinite time, $\mathrm{k}$ and $\lambda$ are model parameters that influence the model curvature, and the time delay factor $\delta$ has also been introduced to make the family of curves more general and to 
take into account a (variable) starting time for the consolidation phase. Notice that the deformation is equal to zero in the model (3) in correspondence to the time: $\hat{t}=\delta$. By using such a model, and depending on the model parameters' values, most of the ground displacement in reclamation areas is expected to principally occur during the first years after the completion of the reclamation procedure, while a residual deformation signal can continue to occur in reclaimed areas, even for a few decades after the end of reclamation, although with very short rates.

By profiting from highly accurate IPTA/SBAS DInSAR products corresponding to previously identified sets of very coherent targets in Types A and B reclamation platforms (see Section III), we preliminarily tested the accordance between the considered model (3), which is suitable for the Shanghai area due to its soil characteristics [54]-[61], and the measured DInSAR-based deformation values.

\section{A. Fitting of the Geotechnical Model With DInSAR-Based Deformation}

For model fitting, we used about 450 points located in the Types A and B areas. These points were identified by searching for the very accurate PS/DS pixels located in the two reclaimed platforms, as shown in Fig. 4, characterized by an (measured) IPTA/SBAS velocity difference of less than $1 \mathrm{~mm} / \mathrm{year}$ and an IPTA/SBAS standard deviation difference less than $5 \mathrm{~mm}$. These types of pixels are shown in Fig. 8(I). The model fitting operation was carried out by considering the four model parameters $S_{m}, k, \lambda$, and $\delta$ of (3), and for each coherent PS/DS target, by searching for the best-fit curve in the least-squares sense ${ }^{2}$ between the model and the (measured) DInSAR-based deformation. The quality of the nonlinear curve fitting was checked by calculating the standard deviation of the difference between the DInSAR time-series and the best-fit model. On average, the obtained standard deviation is $1 \mathrm{~mm}$, thus showing good agreement between the two datasets. Note also that the accuracy of DInSAR-based measurements in Type A/Type B regions is equal to $5 \mathrm{~mm}$ (as evaluated in Section IV). Accordingly, the estimated model confidence is globally at about $6 \mathrm{~mm}$. The obtained results are very promising, although they would require further validations to be carried out in future years via additional DInSAR-based analyses.

By analyzing the retrieved displacement models, we obtained some preliminary predictions about the expected cumulative deformation (calculated with respect to the first SAR acquisition of February 26, 2007) in 2015, 2020, and 2025, as depicted in Fig. 8(I)-(III). As evident, the eastern areas (where reclamation procedures started more recently) are expected to be subject in the near future to larger and more long-lasting deformations than in the western sectors. From these models, we

\footnotetext{
${ }^{2}$ Note that DInSAR-based measurements are still calculated with respect to the first SAR acquisition time. However, at that time the consolidation phase was still active, so we searched for the correct time delay of the family of curves in (3) to best-fit the model and DInSAR measurements.
}
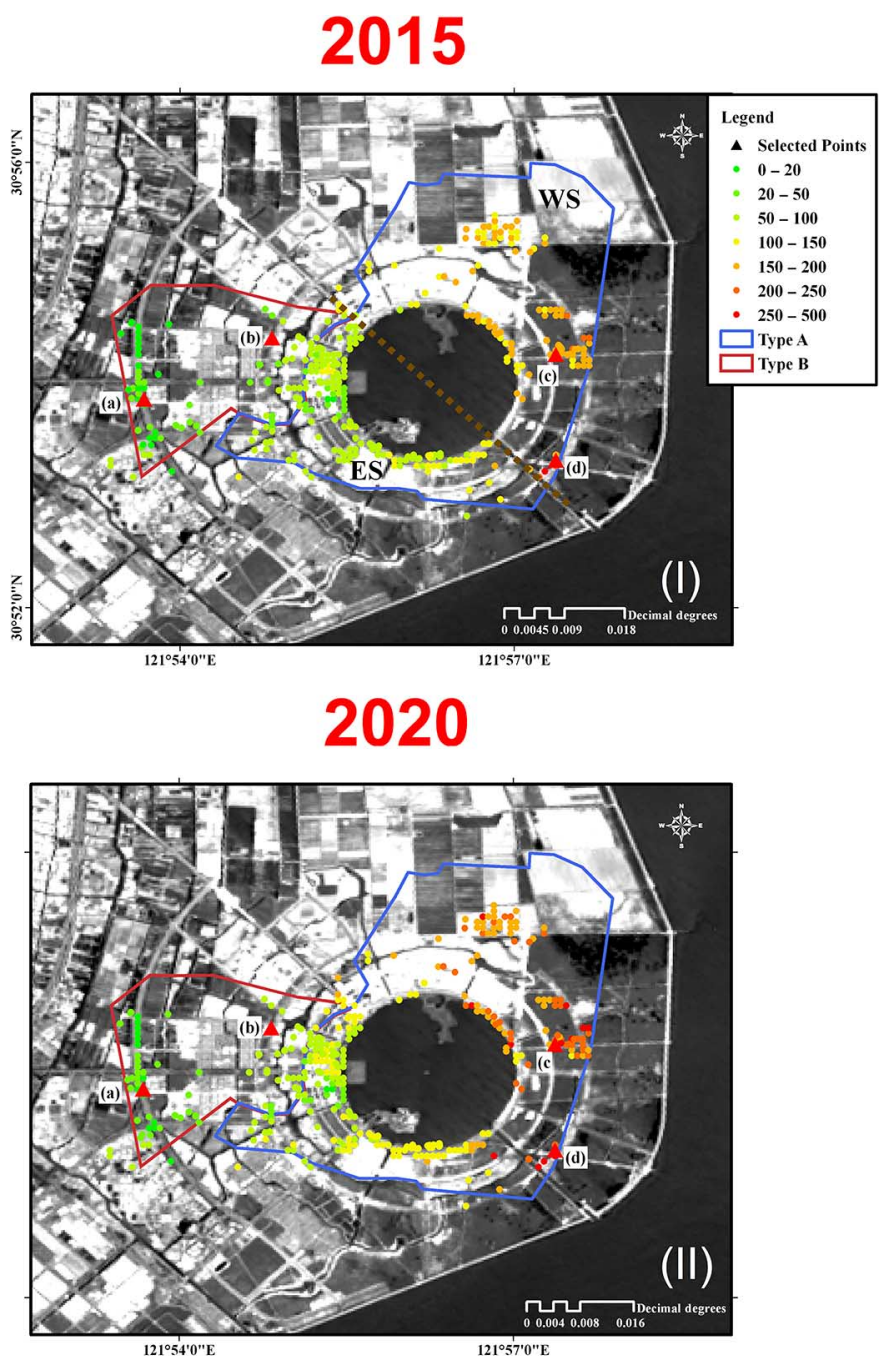

2025

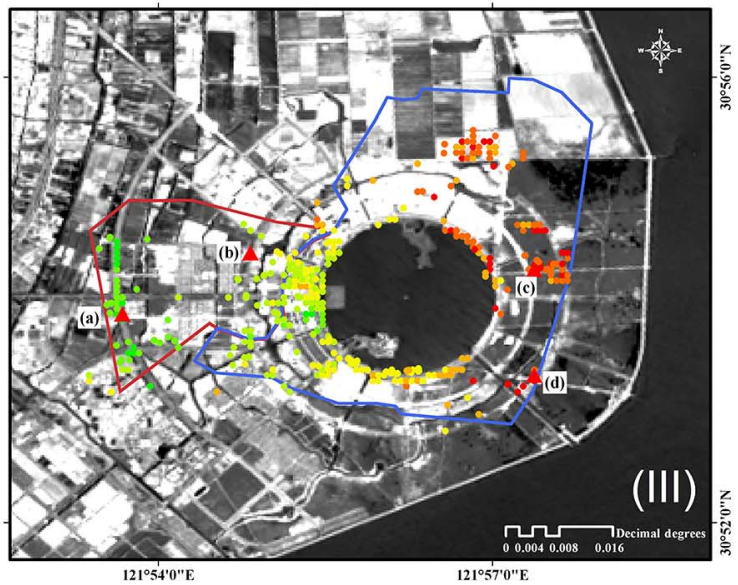

Fig. 8. Cumulative displacement (estimated with respect to the first SAR time acquisition in February 2007) in January for each year of 2015, 2020, and 2025, as retrieved by the prediction models that best fit the PS/DS DInSAR time-series observations. Type A is distinguished in the two East and West subsections, labelled here as ES and WS, respectively. Deformation values are expressed in millimetres. 


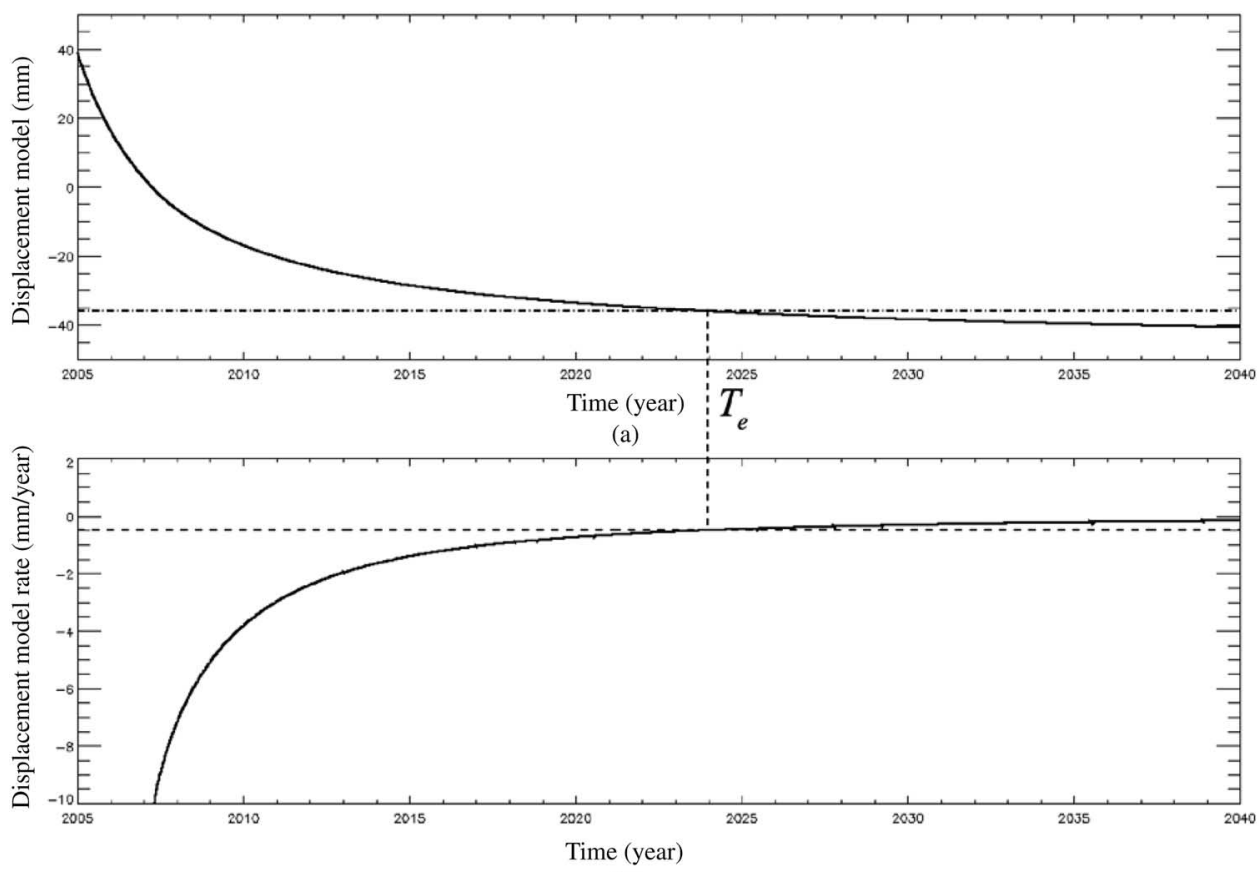

(b)

Fig. 9. Prediction model analysis. (a) Prediction model relevant to the pixel labelled as (a) in Fig. 8. (b) Modelled displacement rate relevant to the same pixel, obtained by simply computing the first derivative of the curve in (a). Note that $\mathrm{T}_{\mathrm{e}}$ is the time in correspondence to which the modelled displacement rate equals $0.5 \mathrm{~mm} / \mathrm{year}$, which in our experiments represents the threshold value corresponding near to the end of the ground consolidation phase.

eventually retrieved the (average) value of the total consolidation time for Types A and B regions, with the aim to globally characterize the present and near future state of the ground settlement consolidation phase in the Nanhui New City.

\section{B. Consolidation Time Estimation}

As a final experiment, we exploited the retrieved best-fit models and figured out the consolidation time at $70 \%, 80 \%$, and $90 \%$ of the expected, model-based total consolidation settlement for all the selected points in Types A and B regions. To achieve this task, we adopted the following strategy. We considered the best-fit model that corresponded to the location of a given PS/DS target on the reclaimed areas, depending on the four model parameters $S_{m}, k, \lambda$, and $\delta$. We assumed that the ground settlement consolidation phase was completed when the (expected) residual deformation rate was less than $0.5 \mathrm{~mm} /$ year. Accordingly, we evaluated the model displacement rate by calculating the first derivative $s_{C M T}^{\prime}(t)$ of the obtained time-varying model. As an example, see Fig. 9(a) and (b), which is relevant to pixel (a) in Fig. 8 located in the Type B region. Reclamation procedures in Type A/Type B regions in Nanhui New City [see Fig. 1(c)] reached its completion between 2004 and 2006. However, given we did not have precise information about the (true) reclamation end time, we imposed in our analysis that, on average, completion time was reached for both the regions on January 2005. We then estimated the duration of consolidation time with respect to that date. However, it is worth remarking that neither the end time of consolidation phase nor the shapes of the best-fit models depend on the exact knowledge of reclamation completion time, but only on the consolidation time duration itself. Accordingly, the end time of the consolidation phase was estimated as follows:

$$
T_{e}: s_{C M T}^{\prime}\left(T_{e}\right)=0 .
$$

Finally, "rough" estimates of the x-percentage total consolidation time duration was obtained as follows:

$$
T_{e}^{(x)}: s_{C M T}\left(T_{e}^{(x)}\right)=\frac{x}{100}\left[s_{C M T}\left(T_{e}\right)-s_{C M T}(0)\right] .
$$

Best-fit models, as well as end times of consolidation phases for the four point targets labelled as (a)-(d) in Fig. 8, located in Type A/Type B regions, have been evaluated and are shown in Fig. 10. We also estimated the values of the standard deviation between the model and the DInSAR-based measurements for the four pixels (a)-(d), reported in Fig. 10.

We first analyzed the consolidation time of duration relevant to the Type B area (see Table V). Our results indicate that $70 \%$ of the consolidation settlement was completed 5 years after the completion of construction, corresponding (approximately) to 2010. In addition, $90 \%$ of the settlement is thought to have occurred after about 9 years. This is in agreement with what was expected in a previous study conducted in the Type B regions [80] suggesting that in 2014, the Type B area should be nearly stable. We anticipate verification of the validity of these affirmations in future studies, as soon as the archive of SAR images acquired by the COSMO-SkyMed [81] sensors' constellation are large enough to permit us to carry out a "sound" DInSAR analysis.

Type A area was reclaimed after Type B area. Thus, Type A takes longer $\mathrm{x}$-percentage total consolidation time than does Type B (See Table V). The average total consolidation time for Type A typically ranges from about 10 to 20 years. However, 


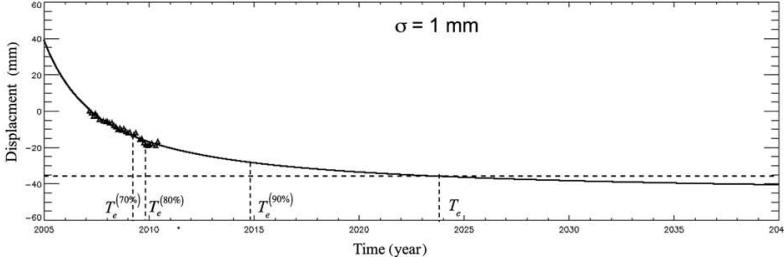

(a)

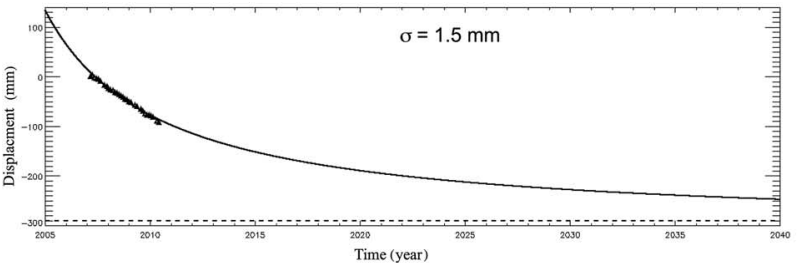

(c)

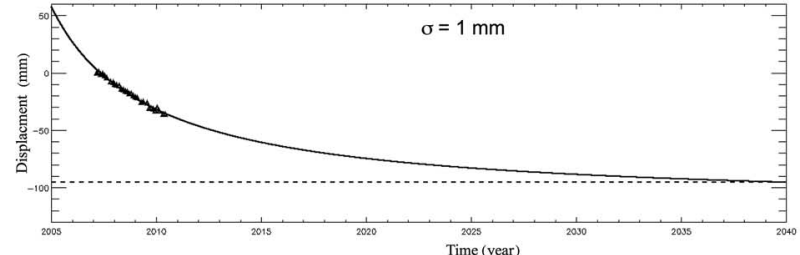

(b)

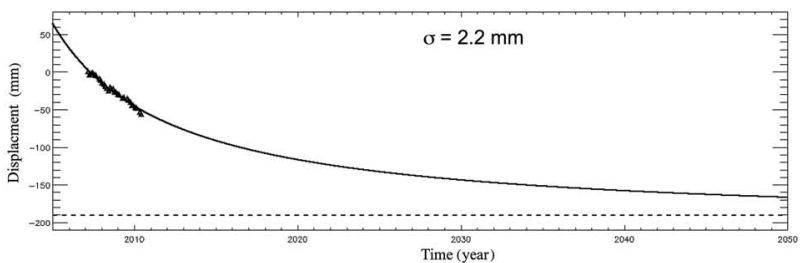

(d)

Fig. 10. Comparison between SBAS (black stars) and model-predicted (continuous lines) best-fit (vertical) displacement time-series, evaluated in correspondence to the four points labelled as (a)-(d) in Fig. 8. We also highlight in (a) the times corresponding to $70 \%$, 80\%, and $90 \%$ of the entire reclamation ground consolidation, estimated by assuming that reclamation processes were nearly completed in January 2005. The standard deviation values between the best-fit model and SBAS deformation time-series relevant to the four DS targets are also indicated. Note also that in correspondence to points (a)-(d), IPTA and SBAS time-series are nearly identical since those pixels belong to the cluster of high-quality points. For this reason, we have only shown the SBAS time-series.

TABLE V

Estimated Total Consolidation Times in REClaimed REgions

\begin{tabular}{|c|c|c|c|}
\hline $\begin{array}{l}\text { Consolidation } \\
\text { degree } \\
\text { x-percentage total } \\
\text { consolidation time (year) }\end{array}$ & $70 \%$ & $80 \%$ & $90 \%$ \\
\hline Type A area & 9.8 & 13.8 & 20.4 \\
\hline Type B area & 5 & 6.7 & 9.2 \\
\hline Western (WS) Type A area & 7.8 & 10.9 & 15.7 \\
\hline Eastern (ES) Type A area & 13.4 & 19.3 & 29.2 \\
\hline
\end{tabular}

the selected points located in the eastern sector of the Type A region, which are adjacent to the coastline under reclaiming, are more critical with respect to the ones located in the central sections of the Type A area. For this reason, we split the entire Type A area into two sectors for our analysis, and estimated the average of the $\mathrm{x}$-percentage total consolidation time for the eastern sectors (ES) and western sectors (WS) separately. As shown in Table $\mathrm{V}$, the average values measured in the WS are less dispersed and range from about 8 to 15 years, indicating that this sector will be stable in about 10 years from the completion of reclamation. Alternatively, the average values measured for the ES have greater fluctuation and range from 13 to 29 years. This is due to the fact that the reclamation of the ES was not completely finished in 2010 .

With respect to previous studies conducted in other reclaimed areas (i.e., Hong Kong International Airport), our results suggest that the consolidation stage will take more time. We believe this is reasonable because the settlement of fill material after land reclamation depends on the type of fill, the method of placement, and the use of the filled area. Moreover, the presented models only concentrate on the consolidation settlement of dredger fill, and do not take into account the presence 
of other potential additional deformation signals due to, for instance, the load of buildings and/or other deformation sources present in the investigated area.

\section{CONCLUSION}

We present, in this work, the results of a study aimed at combining DInSAR-based displacement observations with one geotechnical model to gain insight about the present and future evolution of the ground consolidation phase in coastal reclaimed areas of the Shanghai megacity. We predominantly focused our analysis on two reclaimed zones in Nanhui New City, which are characterized by inherently different dredger fills. A cross-comparison analysis between DInSAR products derived by applying the SBAS and the PS-IPTA processing chain to a set of ENVISAT SAR images has allowed us to identify a grid of about 400 measurement points in reclaimed areas. The results of this analysis showed that the accuracy of the DInSAR displacement measurements is very high. The PSSBAS cross-comparison investigation, which represents one of the first attempts specifically carried out in reclaimed areas, verified that the average PS/SBAS LOS velocity difference over reclaimed platforms is approximately $1.2 \mathrm{~mm} /$ year. The relevant standard deviation of the difference time-series is approximately $5 \mathrm{~mm}$, despite the increased level of noise due to temporal decorrelation artifacts. These numbers suggest that during the first stages of the completion of the reclamation processes, both PS and SB DInSAR methods were able to identify clusters of points that preserve high levels of accuracy. As a result, we obtained a three-dimensional (3-D) space/time characterization of the on-going deformations that affect the reclaimed platforms. Furthermore, by using a population of about 400 high-quality measurements points, we were able to test the relation between DInSAR observations and geotechnical models of the displacement time-evolution. As a result, we eventually recovered some predictions regarding the time of ground consolidation completion in the two identified areas subject to reclamation over recent years, thus obtaining rough estimates of the predicted time duration. Overall, the results of our investigation are very promising; however, they require additional improvement and confirmation through future analyses as soon as additional data are available to better constrain the model with data. Fortunately, the availability of a large set of new SAR data acquired by the COSMO-SkyMed constellation over the Shanghai basin, characterized by improved spatial and temporal resolutions with respect to the ENVISAT sensor, will be profitable for the achievement of this task.

\section{ACKNOWLEDGMENT}

The authors thank ESA for providing the SAR ENVISAT data from the Cat-1 Project \#11461. They also kindly thank the University of Delft, The Netherlands, for the related and precise orbits. The DEM of the investigated zones was acquired through the SRTM archive. Moreover, They would like to thank R. Lanari and M. Manunta for their help and support within the Dragon III project and for the accomplishments of the current investigation. Finally, They are grateful to L. Richards for language polishing, the editor and two anonymous reviewers for their valuable remarks and suggestions.

\section{REFERENCES}

[1] R. Bürgmann, P. A. Rosen, and E. J. Fielding, "Synthetic aperture radar interferometry to measure Earth's surface topography and its deformation," Аnnu. Rev. Earth Planet. Sci., vol. 28, pp. 169-209, May 2000.

[2] D. Massonnet and K. L. Feigl, "Radar interferometry and its application to changes in the Earth's surface," Rev. Geophys., vol. 36, pp. 441-500, 1998.

[3] D. Massonnet et al., "The displacement field of the Landers earthquake mapped by radar interferometry," Nature, vol. 364, pp. 138-142, Jul. 1993.

[4] D. Massonnet, P. Briole, and A. Arnaud, "Deflation of Mount Etna monitored by spaceborne radar interferometry," Nature, vol. 375, pp. 567-570, Jun. 1995.

[5] G. Peltzer and P. A. Rosen, "Surface displacement of the 17 May 1993 Eureka Valley earthquake observed by SAR interferometry," Science, vol. 268, pp. 1333-1336, Jun. 1995.

[6] Y. Fialko, M. Simons, and D. Agnew, "The complete (3-D) surface displacement field in the epicentral area of the $1999 \mathrm{Mw} 7.1$ Hector Mine earthquake, California, from space geodetic observations," Geophys. Res. Lett., vol. 28, pp. 3063-3066, Aug. 2001.

[7] E. Rignot, "Fast recession of a west Antarctic glacier," Science, vol. 281, pp. 549-551, 1998.

[8] A. Ferretti, C. Prati, and F. Rocca, "Permanent scatterers in SAR interferometry," IEEE Trans. Geosci. Remote Sens., vol. 39, no. 1, pp. 8-20, Jan. 2001.

[9] C. Werner, U. Wegmüller, T. Strozzi, and A. Wiesmann, "Interferometric point target analysis for deformation mapping," in Proc. IEEE Int. Geosci. Remote Sens. Symp. (IGARSS'03), Toulouse, France, Jul. 21-25, 2003, vol. 7, pp. 4362-4364.

[10] A. Hooper, H. Zebker, P. Segall, and B. M. Kampes, "A new method for measuring deformation on volcanoes and other natural terrains using InSAR persistent scatterers," Geophys. Res. Lett., vol. 31, no. 23, p. L23611, Dec. 2004.

[11] B. M. Kampes, Radar Interferometry: Persistent Scatterer Technique. New York, NY, USA: Springer, 2006.

[12] P. Berardino, G. Fornaro, R. Lanari, and E. Sansosti, "A new algorithm for surface deformation monitoring based on small baseline differential SAR interferograms," IEEE Trans. Geosci. Remote Sens., vol. 40, no. 11, pp. 2375-2383, Nov. 2002.

[13] S. Usai, "A least squares database approach for SAR interferometric data," IEEE Trans. Geosci. Remote Sens., vol. 41, no. 4, pp. 753-760, Apr. 2003.

[14] O. Mora, J. J. Mallorquí, and A. Broquetas, "Linear and nonlinear terrain deformation maps from a reduced set of interferometric SAR images," IEEE Trans. Geosci. Remote Sens., vol. 41, no. 10, pp. 2243-2253, Oct. 2003.

[15] M. Crosetto, B. Crippa, and E. Biescas, "Early detection and in-depth analysis of deformation phenomena by radar interferometry," Eng. Geol., vol. 79, no. 1/2, pp. 81-91, Jun. 2005.

[16] K. A. C. de Macedo et al., "Long-term airborne DInSAR measurements at X- and P-bands: A case study on the application of surveying geohazard threats to pipelines," IEEE J. Sel. Topics Appl. Earth Observ. Remote Sens., vol. 5, no. 3, pp. 990-1005, Jun. 2012.

[17] A. Hooper, "A multi-temporal InSAR method incorporating both persistent scatterer and small baseline approaches," Geophys. Res. Lett., vol. 35, no. 16, p. L16302, Aug. 2008.

[18] H. A. Zebker and J. Villasenor, "Decorrelation in interferometric radar echoes," IEEE Trans. Geosci. Remote Sens., vol. 30, no. 5, pp. 950-959, Sep. 1992.

[19] G. Franceschetti and R. Lanari, Synthetic Aperture Radar Processing. Boca Raton, FL, USA: CRC Press, Mar. 1999.

[20] G. Strang, Linear Algebra and Its Appications. Orlando, FL, USA: Harcourt Brace Jovanovich, 1988.

[21] R. Lanari et al., "A small baseline approach for investigating deformation on full resolution differential SAR interferograms," IEEE Trans. Geosci. Remote Sens., vol. 42, no. 7, pp. 1377-1386, Jul. 2004.

[22] S. Stramondo et al., "Advanced DInSAR analysis on mining areas: La Union case study (Murcia, SE Spain)," Eng. Geol., vol. 90, no. 3-4, pp. 148-159, Mar. 2007. 
[23] A. Hooper, P. Segall, and H. Zebker, "Persistent scatterer interferometric synthetic aperture radar for crustal deformation analysis, with application to Volcan Alcedo, Galapagos," J. Geophys. Res., vol. 112, no. B7, p. B07407, Jul. 2007.

[24] P. Teatini et al., "Assessing short- and long-time displacements in the Venice coastland by synthetic aperture radar interferometric point target analysis," Geophys. Res., vol. 112, no. F1, p. F01012, Mar. 2007.

[25] T. R. Lauknes et al., "Detailed rockslide mapping in northern Norway with small baseline and persistent scatterer interferometric SAR time series method," Remote. Sens. Environ., vol. 114, no. 9, pp. 2097-2109, Sep. 2010.

[26] V. Tofani, F. Raspini, F. Catani, and N. Casagli, "Persistent scatterer interferometry (PSI) technique for landslide characterization and monitoring," Remote. Sens., vol. 5, no. 3, pp. 1045-1065, Mar. 2013.

[27] U. Wegmueller, D. Walter, V. Spreckels, and C. L. Werner, "Nonuniform ground motion monitoring with TerraSAR-X persistent scatterer interferometry," IEEE Trans. Geosci. Remote Sens., vol. 48, no. 2, pp. 895-904, Feb. 2010.

[28] E. Sansosti, F. Casu, M. Manzo, and R. Lanari, "Space-borne radar interferometry techniques for the generation of deformation time series: An advanced tool for Earth's surface displacement analysis," Geophys. Res. Lett., vol. 37, no. 20, p. L20305, Oct. 2010.

[29] R. Lanari et al., "An overview of the small baseline subset algorithm: A DInSAR technique for surface deformation analysis," Pure Appl. Geophys., vol. 164, no. 4, pp. 637-661, Jan. 2007.

[30] M. Manzo, Y. Fialko, F. Casu, A. Pepe, and R. Lanari, "A quantitative assessment of DInSAR measurements of interseismic deformation: The southern san andreas fault case study," Pure Appl. Geophys., vol. 169, no. 8, pp. 1463-1482, Aug. 2012.

[31] E. Trasatti et al., "The 2004-2006 uplift episode at Campi Flegrei caldera (Italy): Constraints from SBAS-DInSAR ENVISAT data and Bayesian source inference," Geophys. Res. Lett., vol. 35, no. 7, p. L07308, Apr. 2008.

[32] R. Lanari et al., "Surface displacements associated with the L'Aquila 2009 Mw 6.3 earthquake (Central Italy): New evidence from SBASDInSAR time series analysis," Geophys. Res. Lett., vol. 37, p. L20309, Oct. 2010.

[33] M. Bonano, M. Manunta, A. Pepe, L. Paglia, and R. Lanari, "From previous C-band to new X-band SAR systems: Assessment of the DInSAR mapping improvement for deformation time-series retrieval in urban areas," IEEE Trans. Geosci. Remote Sens., vol. 51, no. 4, pp. 1973-1984, Apr. 2013.

[34] E. Sansosti et al., "How second generation SAR systems are impacting the analysis of ground deformation," Int J. Appl. Earth Obs., vol. 28, no. 1, pp. 1-11, 2014.

[35] B. Osmanoglu, T. H. Dixon, S. Wdowinski, E. Cabral-Cano, and Y. Jiang, "Mexico city subsidence observed with persistent scatterer InSAR," Int. J. Appl. Earth Obs., vol. 13, no. 1, pp. 1-12, Feb. 2011.

[36] L. Tao, H. Zhang, C. Wang, and Y. Tang, "Ground deformation retrieval using quasi coherent targets DInSAR, with application to suburban area of Tianjin, China," IEEE J. Sel. Topics Appl. Earth Observ. Remote Sens., vol. 5, no. 3, pp. 867-873, Jun. 2012.

[37] A. I. Zakharov et al., "Earth surface in the kuznetsk coal basin caused by manmade and natural seismic activity according to ALOS PALSAR interferometry," IEEE J. Sel. Topics Appl. Earth Observ. Remote Sens., vol. 6, no. 3, pp. 1578-1583, Jun. 2013.

[38] S. L. Shen, "Geological environmental character of Lin Gang New City and its influences to the construction," Shanghai Geol., vol. 105, pp. 2428, Dec. 2008 (in Chinese).

[39] R. J. Hoeksema, "Three stages in the history of land reclamation in the Netherlands," Irrig. Drain., vol. 56, pp. 113-216, Dec. 2007.

[40] H. H. Al-Barwani and A. Purnama, "Re-assessing the impact of desalination plants brine discharges on eroding beaches," Desalination, vol. 204, pp. 94-101, Mar. 2007

[41] I. Douglas and N. Lawson, "Airport construction: materials use and geomorphic change," J. Air Transp. Manage., vol. 9, no. 3, pp. 177-185, May 2003.

[42] Q. Zhao et al., "InSAR detection of residual settlement of an ocean reclamation engineering project: A case study of Hong Kong International Airport," J. Oceanogr., vol. 67, no. 4, pp. 415-426, Aug. 2011.

[43] D. Ganesalingam, J. Ameratunga, G. Scweitzer, P. Boyle, and S. Sivakugan, "Anisotropy in the permeability and consolidation characteristics of dredged mud," in Proc. 11th Australia-New Zealand Conf. Geomech. Ground Eng. Changing World (ANZ'12), 2012, pp. $752-757$.

[44] D. R. E. Philip and J. D. McIlquham, "Geotechnical design for the Port Botany expansion project," in Proc. ICE-Geotech. Eng., 2011, pp. $149-167$
[45] A. M. Puzrin, E. E. Alonso, and N. M. Pinyol, "Unexpected excessive settlements: Kansai International Airport, Japan," in Geomechanics of Failures. New York, NY, USA: Springer, 2010, pp. 23-43.

[46] A. Rizzo, "Metro Doha," Cities, vol. 31, pp. 533-543, Apr. 2013.

[47] H. Yang, "Legal regulation of land reclamation in China's coastal area," Coast. Manage., vol. 42, no. 1, pp. 9-79, Jan. 2014.

[48] S. L. Shen, "Geological environmental character of Lin Gang New City and its influences to the construction," Shanghai Geol., vol. 105, pp. 2428, Dec. 2008 (in Chinese).

[49] H. Wang et al., "InSAR reveals coastal subsidence in the Pearl River Delta, China," Geophys. J. Int., vol. 191, no. 3, pp. 1119-1128, Dec. 2012.

[50] F. Casu, M. Manzo, and R. Lanari, "A quantitative assessment of the SBAS algorithm performance for surface deformation retrieval from DInSAR data," Remote Sens. Environ., vol. 102, no. 3/4, pp. 195-210, Jun. 2006.

[51] D. Raucoules et al., "Validation and intercomparison of Persistent Scatterers Interferometry: PSIC4 project results," J. Appl. Geophys., vol. 68, no. 3, pp. 335-347, Jul. 2009.

[52] A. Ferretti et al., "Submillimeter accuracy of InSAR time series: Experimental validation," IEEE Trans. Geosci. Remote Sens., vol. 45, no. 5, pp. 1142-1153, May 2007.

[53] M. Crosetto et al., "Final report of the validation of existing processing chains in Terrafirma stage 2," Terrafirma project, ESRIN/Contract no. 19366/05/I-E, 2008 [Online]. Available: http://www.terrafirma.eu.com/product_validation.htm

[54] P. Yang, Y.-Q. Tang, N.-Q. Zhou, and J.-X. Wang, "Consolidation settlement of Shanghai dredger fill under self-weight using centrifuge modeling test," J. Cent. South Univ. Technol., vol. 39, no. 4, pp. 862-866, Aug. 2008 (in Chinese).

[55] C. Yun, Tu shuo Chang Jiang He Kou Yan Bian. China, Beijing: China Ocean Press, 2010, pp. 20-65 (in Chinese).

[56] K. W. Chan, "Misconceptions and Complexities in the Study of China's Cities: Definitions, statistics, and implications," Eurasian Geogr. Econ., vol. 48, no. 4, pp. 383-412, Aug. 2007.

[57] Shanghai Municipal Bureau of Planning, and Land Resources (SMBPLR), "Shanghai geological environmental bulletin (2001-2009)," Shanghai Mun. Bureau Plan. Land Res., Shanghai, 2009 (In Chinese).

[58] Y. Shi, X. Yan, and N. Zhou, "Land subsidence induced by recent alluvia deposits in Yangtze River delta area, a case study of Shanghai Lingang New City," J. Eng. Geol., vol. 15, no. 3, pp. 391-402, Dec. 2007 (in Chinese).

[59] G. W. Plant, C. S. Covil, and R. A. Hughes, "Geology, surveying, reclamation settlement," in Site Preparation of the New Hong Kong International Airport, 1st ed. London, U.K.: Thomas Telford, 1998, pp. 45, 387-416, 515-517.

[60] S. L. Yang, B. W. Shi, T. J. Bouma, T. Ysebaert, and X. X. Luo, "Wave attenuation at a salt marsh margin: A case study of an exposed coast on the Yangtze Estuary," in Intertidal Deposits: River Mouths, Tidal Flats, and Coastal Lagoons, vol. 16, D. Eisma, Ed., 1st ed. Boca Raton, FL, USA: CRC Press, 1998, pp. 169-182.

[61] Z. Wei, G. Zhai, and X. Yan, Atlas of Shanghai Urban Geology. China, Beijing: Geological Publishing House, 2010, pp. 1-97.

[62] Shanghai Institute of Geological Survey (SIGS), "The geological hazard risk assessment report of Lingang New City (PD7)," Shanghai Inst. Geo. Surv., Shanghai, 2006 (in Chinese).

[63] Shanghai Institute of Geological Survey (SIGS), "The geological hazard risk assessment report of Lingang New City (PD8)," Shanghai Inst. Geo. Surv., Shanghai, 2006 (in Chinese).

[64] Shanghai Institute of Geological Survey (SIGS), "The geological hazard risk assessment report of Lingang New City (PD9)," Shanghai Inst. Geo. Surv., Shanghai, 2006 (in Chinese).

[65] C. Werner, U. Wegmuller, T. Strozzi, and A. Wiesmann, "Gamma SAR and interferometric processing software," in Proc. ESA-ENVISAT Symp., 2000, pp. 16-20.

[66] P. A. Rosen et al., "Synthetic aperture radar interferometry," Proc. IEEE, vol. 88, no. 3, pp. 333-382, Mar. 2000

[67] Y. Yang et al., "A simple solution to mitigate noise effects in timeredundant sequences of small baseline multi-look DInSAR interferograms," Remote Sens. Lett., vol. 4, no. 6, pp. 609-618, Mar. 2013.

[68] A. Pepe, L. D. Euillades, M. Manunta, and R. Lanari, "New advances of the extended minimum cost flow phase unwrapping algorithm for SBASDInSAR analysis at full spatial resolution," IEEE Trans. Geosci. Remote Sens., vol. 49, no. 10, pp. 4062-4079, Oct. 2011.

[69] Y. Yang, A. Pepe, M. Manzo, F. Casu, and R. Lanari, "A regiongrowing technique to improve multi-temporal DInSAR interferogram phase unwrapping performance," Remote Sens. Lett., vol. 4, no. 10, pp. 988-997, Aug. 2013, doi: 10.1080/2150704X.2013.826835. 
[70] F. Casu et al., "SBAS-DInSAR parallel processing for deformation timeseries computation," IEEE J. Sel. Topics Appl. Earth Observ. Remote Sens., vol. 7, no. 8, pp. 3285-3296, Aug. 2014.

[71] A. Pepe and R. Lanari, "On the extension of the minimum cost flow algorithm for phase unwrapping of multitemporal differential SAR interferograms," IEEE Trans. Geosci. Remote Sens., vol. 44, no. 9, pp. 2374-2383, Sep. 2006.

[72] P. Shanker, F. Casu, H. Zebker, and R. Lanari, "Comparison of persistent scatterers and small baseline time-series InSAR results: A case study of the San Francisco bay area," Geosci. Remote Sens. Lett., vol. 8, no. 4, pp. 592-596, 2011.

[73] Y. Yajing et al., "Mexico city subsidence measured by InSAR time series: Joint analysis using PS and SBAS approaches," IEEE J. Sel. Topics Appl. Earth Observ. Remote Sens., vol. 5, no. 4, pp. 1312-1326, Aug. 2012

[74] M.-S. Yang, Y. Jiang, M. Liao, and H. Wang, "The analysis of the subsidence patterns in Lingang New City (Shanghai) using high-resolution SAR images," Shanghai Land Resour., vol. 34, no. 4, pp. 12-16, Jun. 2013 (in Chinese).

[75] Y.-S. Xu, L. Ma, Y.-J. Du, and S.-L. Shen, "Analysis of urbanizationinduced land subsidence in Shanghai," Nat. Hazards, vol. 63, no. 2, pp. 1255-1267, Sep. 2012.

[76] R. N. Taylor, "Centrifuges in modeling: Principles and scale effects," in Geotechnical Centrifuge Technology, Glasgow, U.K.: Blackie Academic and Professional, 1995, pp. 20-30.

[77] Z. D. Cui and Y. Q. Tang, "Land subsidence and pore structure of soils caused by the high-rise building group through centrifuge model test," Eng. Geol., vol. 113, pp. 44-52, Jun. 2010.

[78] Y. Q. Tang, Z. D. Cui, J. X. Wang, L. Yan, and X. Yan, "Application of grey theory-based model to prediction of land subsidence due to engineering environment in Shanghai," Environ. Geol., vol. 55, no. 3, pp. 583-593, Aug. 2008.

[79] K. Been and G. C. Sills, "Self-weight consolidation of soft soils: An experimental and theoretical study," Geotechnique, vol. 31, no. 4, pp. 519-535, 1981.

[80] S. L. Gong, "The study on acting factors and systemic control of land subsidence in Shanghai," Ph.D. dissertation, State Key Lab. Estuarine and Coastal Res., East China Normal Univ., Shanghai, China, Jun. 2008, p. 77 (in Chinese).

[81] F. Covello et al., "COSMO-SkyMed an existing opportunity for observing the Earth," J. Geodyn., vol. 49, no. 3/4, pp. 171-180, Apr. 2010.

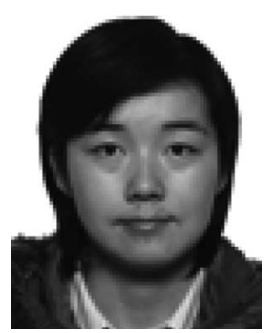

Qing Zhao received the Bachelor's degree in surveying and mapping from the Central South University, Changsha, China, in 2004, the M.Sc. degree in remote sensing and cartography from Wuhan University, Wuhan, China, in 2006, and the Ph.D. degree in geoinformation science from Chinese University of Hong Kong, Hong Kong, China, in 2010.

She joined the Key Laboratory of Geographical Information Science, Ministry of Education, East China Normal University, Shanghai, China, in June 2010. Since January 2014, she has held a position as an Associate Professor with the College of Geography Science, East China Normal University, where she teaches two courses. She is also a Course Instructor with New York University Shanghai, Shanghai, China, in 2015. Her research interests include synthetic aperture radar interferometry, ocean remote sensing, and air quality monitoring and forecasting.

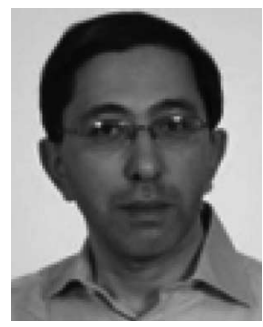

Antonio Pepe (M'12) received the Laurea degree in electronic engineering and the Ph.D. degree in electronic and telecommunication engineering from the University of Napoli Federico II, Napoli, Italy, in 2000 and 2007, respectively.

After graduation, following a short experience with Wind Telecommunication S.p.A., Rome, Italy, he joined the Istituto per il Rilevamento Elettromagnetico dell'Ambiente, Italian National Research Council (CNR), Napoli, Italy, in 2001, where he currently holds a permanent position of
Researcher. He was a Visiting Scientist at University of Texas at Austin, Austin, in 2005, at the Jet Propulsion Laboratory (JPL), Caltech, Pasadena, CA, in 2009, and at the East China Normal University (ECNU), Shanghai in 2014. He acts as reviewer of several peer-reviewed international journals. His research interests include the development of advanced DInSAR algorithms aimed at monitoring surface deformation phenomena induced by subsidence, volcano activities, and earthquakes, with a particular interest toward the phase unwrapping problems. More recently, he has developed research activities for the generation of DInSAR products through new generation SAR instruments and for the generation of mixed ScanSAR-Stripmap DInSAR analyses.

Dr. Pepe is Member of the Editorial Board of the Advances in Geology and Asian Journal of Geoscience journals, Hindawi Publishing Corporation. Since 2012 he has also been an Adjunct Professor of Signal Theory at the Università della Basilicata, Potenza, Italy.

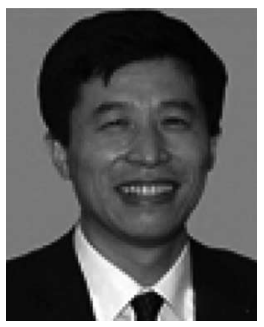

Wei Gao received the Ph.D. degree in applied meteorology and environmental science from Purdue University, West Lafayette, IN, USA, in 1997. He did the Postdoctoral training at the National Centre for Atmospheric Research, Boulder, CO, USA.

$\mathrm{He}$ is a Professor with the College of Geography Science, East China Normal University, Shanghai, China. He is also the Director of the Key Laboratory of Geographical Information Science, Ministry of Education, East China Normal University, Shanghai, China. He has published numerous academic papers and edited numerous books, scientific proceedings, and special journal issues. His research interests include atmospheric radiation and modelling, remote sensing applications, regional climate/ecosystem modeling, geographic information systems, UV radiation, and other climate stress factor influences on ecosystems and their impact on climate change.

Dr. Gao is a Fellow of the International Society for Optical Engineering (SPIE).

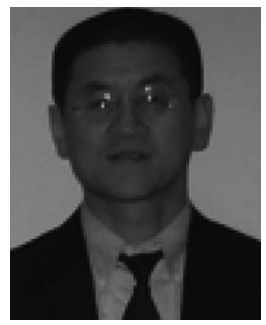

Zhong Lu (S'96-A'97-M'97-SM'07) received the B.S. and M.S. degrees in geophysics from Peking University, Beijing, China, in 1989 and 1992, and the $\mathrm{Ph} . \mathrm{D}$. degree in geophysics from the University of Alaska Fairbanks, Fairbanks, AK, USA, in 1996.

$\mathrm{He}$ was a Physical Scientist with United States Geological Survey (USGS), Vancouver, Washington, during 1997-2013, and is now a Professor and endowed Shuler-Foscue Chair with Huffington Department of Earth Sciences, Southern Methodist University, Dallas, TX, USA (www.smu.edu/dedman/lu). He is a Principal Investigator of projects funded by the National Aeronautics and Space Administration (NASA), European Space Agency, Japan Aerospace Exploration Agency, German Aerospace Agency, and USGS on the study of land surface deformation using satellite interferometric synthetic aperture radar (SAR) (InSAR) imagery. He has produced more than 45 lead-authored and 70 coauthored peer-reviewed journal articles and book chapters focused on InSAR techniques and applications. He has authored a book InSAR Imaging of Aleutian Volcanoes: Monitoring a Volcanic Arc From Space (Springer, April 2014). His research interests include technique developments of SAR, InSAR, and persistent scatterer InSAR processing and their applications on natural hazard monitoring and natural resource management.

Dr. Lu was the Recipient of an American Society for Photogrammetry and Remote Sensing Award for Best Scientific Paper in Remote Sensing, NASA Group Achievement Award, NASA Certificate of Appreciation, Raytheon Distinguished Level Award for Excellence in Technology, Science Applications International Corporation Technical Fellow, and Jerald Cook Memorial Award. $\mathrm{He}$ is a Committee Member of the International User Team for RadarsatC SAR Constellations, the GeoEarthscope InSAR User Working Group, the NASA's Alaska Satellite Facility UserWorking Group, and the upcoming NASA-India SAR Science Definition Team. He is the Chair of Western North America InSAR (WInSAR) Consortium. He is a Member of editorial boards for International Journal of Image and Data Fusion, Geomatics, Natural Hazards and Risk, Dataset Papers in Geosciences, and an Associate Editor for Frontier in Volcanology. 


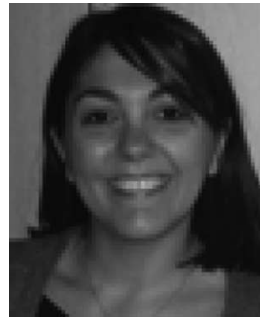

Manuela Bonano received the Laurea degree (summa cum laude) in environmental engineering from the University of Cagliari, Cagliari, Italy, in 2004, and the Ph.D. degree in infrastructures and transportation from the University of Rome "La Sapienza," Rome, Italy, in 2012.

In 2007, she joined the Istituto per il Rilevamento Elettromagnetico dell'Ambiente (IREA-CNR), where she currently holds a Research position. In 2011, she was a Visiting Scientist at the Earth and Planetary Science (EPS) Department, University of California at Berkeley (UCB), Berkeley, CA, USA. Her research interests include the DInSAR field concerning the development of advanced multi-pass interferometry algorithms, particularly focused on full resolution DInSAR analyses for investigating local deformation affecting single buildings and man-made structures. Currently, she works on the development and application of advanced DInSAR techniques for processing SAR data acquired by novel generation satellites, such as X-band COSMO-SkyMed and TerraSAR-X, as well as C-band Sentinel-1.

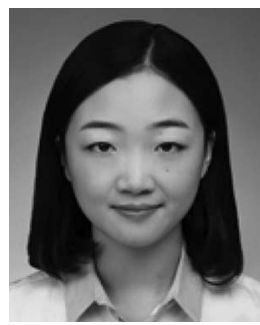

Man L. He received the Bachelor's degree in geographic information system and the M.Sc. degree in cartography and geographic information system from East China Normal University, Shanghai, China, in 2009 and 2012, respectively.

Since 2012, she has been with Shanghai Surveying and Mapping Institute, Shanghai, China, where she currently holds a Research Fellowship position. Her research interests include cartography and geographic information system.

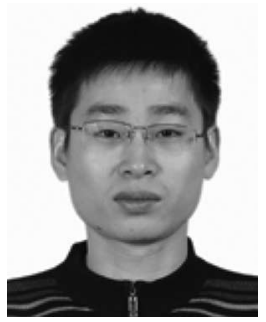

Jun Wang received the Bachelor's degree in hydrology and water resources engineering from China University of Geosciences, Wuhan, China, in 2006. $\mathrm{He}$ is currently pursuing the M.Sc. degree in cartography and geographic information system at the same university.

Since 2007, he has been with Shanghai Surveying and Mapping Institute, Shanghai, China, where he currently holds a Research Fellowship position. His research interests include land resources information engineering.

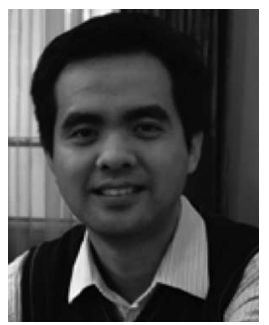

Xi Tang received the Bachelor's and Ph.D. degrees in cartography and geographic information system from East China Normal University, Shanghai, China, in 2005 and 2010, respectively.

After graduation, he joined the Key Laboratory of Geographical Information Science, Ministry of Education, East China Normal University, Shanghai, China. He is an Associate Professor with the College of Geography Science, Key Laboratory of Geographic Information Science, Ministry of Education, East China Normal University. His research interests include thematic mapping, spatial planning cartography, remote sensing imagery mapping for industry and urban planning, and map symbolization. 